\title{
Synthesis of Biaryls via Decarboxylative Pd-Catalyzed Cross-Coupling Reaction
}

\author{
Jean-Michel Becht, ${ }^{\dagger *}$ Cédric Catala, ${ }^{\dagger}$ Claude Le Drian ${ }^{\dagger}$ and Alain Wagner ${ }^{* *}$ \\ ${ }^{\dagger}$ Université de Haute-Alsace, Ecole Nationale Supérieure de Chimie de Mulhouse, \\ Laboratoire de Chimie Organique et Bioorganique, UMR-CNRS 7015, \\ 3 rue Alfred Werner, 68093 Mulhouse Cedex, France \\ * Novalyst Discovery, Bioparc, Boulevard Sébastien Brant BP 30170, 67405 Illkirch Cedex, France
}

\section{SUPPORTING INFORMATIONS}

\section{Table of contents}

General Remarks

General Procedure for the Synthesis and Characterizations of Biaryls 3a-t S2-S5

NMR Spectra of Biaryls 3a-t

S6-S33 


\section{General Remarks.}

The reagents were obtained from commercial sources and were used without further purifications. DMSO was dried on molecular sieves $4 \AA$. The syntheses of biaryls 3a-r were carried out in anhydrous glassware under an atmosphere of argon. Purifications of compounds 3a-r were performed by flash-chromatography on silica gel (40-63 $\mu \mathrm{m}$, Merck). ${ }^{1} \mathrm{H}$ and ${ }^{13} \mathrm{C}$ NMR spectra were recorded using a $400 \mathrm{MHz}$ instrument in $\mathrm{CDCl}_{3}$. Chemical shifts are reported in parts per million ( $\delta$ ) downfield from TMS. Spin multiplicities are indicated by the following symbol: s (singlet), d (doublet), $\mathrm{t}$ (triplet), q (quadruplet), hept (heptuplet) and $\mathrm{m}$ (multiplet). IR spectra were recorded on a Nicolet 205 FT-IR spectrometer. 2,6-Diisopropoxybenzoic acid was prepared according to a previous literature report. ${ }^{1}$ Compounds $3 \mathbf{3 a}, \mathbf{3 b}, \mathbf{3 f}, \mathbf{3 g}, \mathbf{3 i}, \mathbf{3 j}, \mathbf{3 k}, \mathbf{3 m}, \mathbf{3 n}, \mathbf{3 o}, \mathbf{3 p}, \mathbf{3 s}$ and $3 \mathbf{t}$ were previously described in the literature. ${ }^{2}$

\section{General Procedure for the Syntheses of Biaryls 3a-t.}

DMSO $(6 \mathrm{~mL})$ was added to a mixture of the aryl iodide $(0.50 \mathrm{mmol}, 1.0$ equiv), the arene carboxylic acid (0.65 mmol, 1.3 equiv), $\mathrm{Ag}_{2} \mathrm{CO}_{3}$ (1.50 mmol, $414 \mathrm{mg}, 3.0$ equiv), $\mathrm{AsPh}_{3}(0.30$ mmol, $91.9 \mathrm{mg}, 0.6$ equiv) and $\mathrm{PdCl}_{2}(0.15 \mathrm{mmol}, 26.6 \mathrm{mg}, 0.3$ equiv). The reaction mixture was degased twice with argon and directly heated at $150{ }^{\circ} \mathrm{C}$ for $6 \mathrm{~h}$. After cooling to rt, the reaction mixture was filtered with Celite and AcOEt $(100 \mathrm{~mL})$ was added to the filtrate. The organic phase was washed with saturated $\mathrm{NH}_{4} \mathrm{Cl}(70 \mathrm{~mL})$, dried with $\mathrm{MgSO}_{4}$, filtered and concentrated under vacuum. The residue was purified by flash-chromatography on silica gel to afford pure biaryls after drying under vacuum (0.1 mbar).

2,4',6-Trimethoxybiphenyl (3a): Elution with AcOEt / Cyclohexane 4:96 afforded $110 \mathrm{mg}$ (90\% yield) of a white solid; mp $114-116{ }^{\circ} \mathrm{C}$ (lit. mp 116-117 ${ }^{\circ} \mathrm{C}$ ). ${ }^{2 \mathrm{a}}{ }^{1} \mathrm{H}$ NMR $\left(400 \mathrm{MHz}, \mathrm{CDCl}_{3}\right) \delta$ (ppm): $3.77(\mathrm{~s}, 6 \mathrm{H}), 3.87(\mathrm{~s}, 3 \mathrm{H}), 6.68\left(\mathrm{~d},{ }^{3} J(\mathrm{H}, \mathrm{H})=8.3 \mathrm{~Hz}, 2 \mathrm{H}\right), 6.98\left(\mathrm{~d},{ }^{3} J(\mathrm{H}, \mathrm{H})=8.8 \mathrm{~Hz}, 2 \mathrm{H}\right)$, $7.31(\mathrm{~m}, 3 \mathrm{H})$.

2,4,4',6-Tetramethoxybiphenyl (3b): Elution with AcOEt / Cyclohexane 10:90 afforded $103 \mathrm{mg}$ (75\% yield) of a white solid; mp 101-103 ${ }^{\circ} \mathrm{C}$ (lit. mp 102-103 $\left.{ }^{\circ} \mathrm{C}\right) .{ }^{2 \mathrm{~b}}{ }^{1} \mathrm{H} \mathrm{NMR}\left(400 \mathrm{MHz}, \mathrm{CDCl}_{3}\right) \delta$ (ppm): 3.74 (s, 6H), 3.84 (s, 3H), 3.87 (s, 3H), 6.24 (s, 2H), $6.94\left(\mathrm{~d},{ }^{3} J(\mathrm{H}, \mathrm{H})=8.8 \mathrm{~Hz}, 2 \mathrm{H}\right), 7.27$ (d, $\left.{ }^{3} J(\mathrm{H}, \mathrm{H})=8.8 \mathrm{~Hz}, 2 \mathrm{H}\right)$.

3-Bromo-2,4',6-trimethoxybiphenyl (3c): Elution with AcOEt / Cyclohexane 5:95 afforded 105 $\mathrm{mg}\left(65 \%\right.$ yield) of a white solid; mp 78-80 ${ }^{\circ} \mathrm{C} .{ }^{1} \mathrm{H}$ NMR $\left(400 \mathrm{MHz}, \mathrm{CDCl}_{3}\right) \delta$ (ppm): 3.40 (s, 3H), $3.74(\mathrm{~s}, 3 \mathrm{H}), 3.87(\mathrm{~s}, 3 \mathrm{H}), 6.67\left(\mathrm{~d},{ }^{3} J(\mathrm{H}, \mathrm{H})=9.1 \mathrm{~Hz}, 1 \mathrm{H}\right), 6.97\left(\mathrm{~d},{ }^{3} J(\mathrm{H}, \mathrm{H})=8.9 \mathrm{~Hz}, 2 \mathrm{H}\right), 7.34(\mathrm{~d}$,

$\left.{ }^{3} J(\mathrm{H}, \mathrm{H})=8.9 \mathrm{~Hz}, 2 \mathrm{H}\right), 7.47\left(\mathrm{~d},{ }^{3} J(\mathrm{H}, \mathrm{H})=9.1 \mathrm{~Hz}, 1 \mathrm{H}\right) .{ }^{13} \mathrm{C} \mathrm{NMR}\left(80 \mathrm{MHz}, \mathrm{CDCl}_{3}\right) \delta(\mathrm{ppm}): 55.2$, $56.1,60.2,108.3,108.7,113.3,114,1,125.3,127.7,131.5,131.6,155.3,157.3,158.7$. IR $\left(\mathrm{CHCl}_{3}\right) v$ 
$\left(\mathrm{cm}^{-1}\right)$ : 3002, 2999, 2935, 1610, 1579, 1516, 1463, 1396, 1286, 1244, 1221, 1088, 1043, 812. Anal. Calcd for $\mathrm{C}_{15} \mathrm{H}_{15} \mathrm{BrO}_{3}$ : C 55.75, $\mathrm{H}$ 4.68, $\mathrm{Br} 24.72$, found: C 55.86, H 4.64, Br 24.79.

2,6-Diisopropoxy-4'-methoxybiphenyl (3d): Elution with AcOEt / Cyclohexane 2:98 afforded 90 mg (62\% yield) of a colorless oil. ${ }^{1} \mathrm{H}$ NMR $\left(400 \mathrm{MHz}, \mathrm{CDCl}_{3}\right) \delta(\mathrm{ppm}): 1.16\left(\mathrm{~d},{ }^{3} J(\mathrm{H}, \mathrm{H})=6.1 \mathrm{~Hz}\right.$, $12 \mathrm{H}), 3.86(\mathrm{~s}, 3 \mathrm{H}), 4.29\left(\mathrm{hept},{ }^{3} J(\mathrm{H}, \mathrm{H})=6.1 \mathrm{~Hz}, 2 \mathrm{H}\right), 6.63\left(\mathrm{~d},{ }^{3} J(\mathrm{H}, \mathrm{H})=8.1 \mathrm{~Hz}, 2 \mathrm{H}\right), 6.90(\mathrm{~d}$, $\left.{ }^{3} J(\mathrm{H}, \mathrm{H})=8.8 \mathrm{~Hz}, 2 \mathrm{H}\right), 7.16\left(\mathrm{t},{ }^{3} J(\mathrm{H}, \mathrm{H})=8.1 \mathrm{~Hz}, 1 \mathrm{H}\right), 7.29\left(\mathrm{~d},{ }^{3} J(\mathrm{H}, \mathrm{H})=8.8 \mathrm{~Hz}, 2 \mathrm{H}\right) .{ }^{13} \mathrm{C} \mathrm{NMR}(80$ $\left.\mathrm{MHz}, \mathrm{CDCl}_{3}\right) \delta$ (ppm): 22.1, 55.1, 71.2, 108.7, 112.5, 122.9, 126.8, 127.7, 132.3, 156.4, 157.8. IR $\left(\mathrm{CHCl}_{3}\right) \vee\left(\mathrm{cm}^{-1}\right): 3006,2976,2936,1590,1517,1459,1383,1243,1176,1115,1061,830$. HRMS: $\mathrm{m} / \mathrm{z}$ calcd for $\mathrm{C}_{19} \mathrm{H}_{24} \mathrm{O}_{3}\left(\mathrm{M}^{+}\right): 300.1725$, found: 300.1719 .

4,4',5'-Trimethoxy-2-nitrobiphenyl (3e): Elution with AcOEt / Cyclohexane 15:85 afforded 114 mg $\left(79 \%\right.$ yield) of a yellow oil. ${ }^{1} \mathrm{H}$ NMR $\left(400 \mathrm{MHz}, \mathrm{CDCl}_{3}\right) \delta(\mathrm{ppm}): 3.86$ (s, 3H), 3.96 (s, 3H), $3.98(\mathrm{~s}, 3 \mathrm{H}), 6.78(\mathrm{~s}, 1 \mathrm{H}), 6.95\left(\mathrm{~d},{ }^{3} J(\mathrm{H}, \mathrm{H})=8.8 \mathrm{~Hz}, 2 \mathrm{H}\right), 7.23\left(\mathrm{~d},{ }^{3} J(\mathrm{H}, \mathrm{H})=8.8 \mathrm{~Hz}, 2 \mathrm{H}\right), 7.52(\mathrm{~s}$, 1H). ${ }^{13} \mathrm{C} \mathrm{NMR}\left(80 \mathrm{MHz}, \mathrm{CDCl}_{3}\right) \delta(\mathrm{ppm}): 55.3,56.3,56.4,107.8,113.6,114.0,129.2,130.4$, 131.0, 141.1, 147.8, 152.1, 159.4. IR $\left(\mathrm{CHCl}_{3}\right) \vee\left(\mathrm{cm}^{-1}\right): 3002,2937,2922,1610,1582,1504,1460$, 1439, 1337, 1284, 1237, 1216, 1180, 1022. HRMS: m/z calcd for $\mathrm{C}_{15} \mathrm{H}_{15} \mathrm{NO}_{5}\left(\mathrm{M}^{+}\right)$: 289.0950, found: 289.0944 .

4'-Methoxy-2-nitrobiphenyl (3f): Elution with AcOEt / Cyclohexane 2:98 afforded 72 mg (63\% yield) of a yellow oil. ${ }^{1} \mathrm{H}$ NMR (400 MHz, $\left.\mathrm{CDCl}_{3}\right) \delta(\mathrm{ppm}): 3.86(\mathrm{~s}, 3 \mathrm{H}), 6.96\left(\mathrm{~d},{ }^{3} \mathrm{~J}(\mathrm{H}, \mathrm{H})=8.6 \mathrm{~Hz}\right.$, $2 \mathrm{H}), 7.26(\mathrm{~m}, 2 \mathrm{H}), 7.45(\mathrm{~m}, 2 \mathrm{H}), 7.60\left(\mathrm{t},{ }^{3} J(\mathrm{H}, \mathrm{H})=8.2 \mathrm{~Hz}, 1 \mathrm{H}\right), 7.81\left(\mathrm{~d},{ }^{3} J(\mathrm{H}, \mathrm{H})=8.1 \mathrm{~Hz}, 1 \mathrm{H}\right)$.

2,3,4,5,6-Pentafluoro-4'-methoxybiphenyl (3g): Elution with AcOEt / Cyclohexane 4:96 afforded $126 \mathrm{mg}$ (92\% yield) of a white solid; mp 116-118 ${ }^{\circ} \mathrm{C} .{ }^{1} \mathrm{H}$ NMR (400 MHz, $\left.\mathrm{CDCl}_{3}\right) \delta$ (ppm): 3.88 $(\mathrm{s}, 3 \mathrm{H}), 7.02\left(\mathrm{~d},{ }^{3} J(\mathrm{H}, \mathrm{H})=8.8 \mathrm{~Hz}, 2 \mathrm{H}\right), 7.37\left(\mathrm{~d},{ }^{3} J(\mathrm{H}, \mathrm{H})=8.8 \mathrm{~Hz}, 2 \mathrm{H}\right)$.

2-Chloro-6-fluoro-4'-methoxybiphenyl (3h): Elution with AcOEt / Cyclohexane 2:98 afforded 97 mg $\left(82 \%\right.$ yield) of a colorless oil. ${ }^{1} \mathrm{H}$ NMR $\left(400 \mathrm{MHz}, \mathrm{CDCl}_{3}\right) \delta(\mathrm{ppm}): 3.87(\mathrm{~s}, 3 \mathrm{H}), 7.00(\mathrm{~d}$, $\left.{ }^{3} J(\mathrm{H}, \mathrm{H})=8.8 \mathrm{~Hz}, 2 \mathrm{H}\right), 7.07\left(\mathrm{t},{ }^{3} J(\mathrm{H}, \mathrm{H})=9.1 \mathrm{~Hz}, 1 \mathrm{H}\right), 7.35(\mathrm{~m}, 4 \mathrm{H}) .{ }^{13} \mathrm{C} \mathrm{NMR}\left(80 \mathrm{MHz}, \mathrm{CDCl}_{3}\right) \delta$ (ppm): 55.2, 113.6, $114.2\left(\mathrm{~d},{ }^{2} J(\mathrm{C}, \mathrm{F})=19.1 \mathrm{~Hz}\right), 124.7,125.3\left(\mathrm{~d},{ }^{4} J(\mathrm{C}, \mathrm{F})=2.8 \mathrm{~Hz}\right), 128.7(\mathrm{~d}$, $\left.{ }^{2} J(\mathrm{C}, \mathrm{F})=7.9 \mathrm{~Hz}\right), 131.4,134.6\left(\mathrm{~d},{ }^{3} J(\mathrm{C}, \mathrm{F})=2.8 \mathrm{~Hz}\right), 160.5\left(\mathrm{~d},{ }^{1} J(\mathrm{C}, \mathrm{F})=196.6 \mathrm{~Hz}\right), 159.4 . \mathrm{IR}$ $\left(\mathrm{CHCl}_{3}\right) \vee\left(\mathrm{cm}^{-1}\right): 3003,2936,2914,1612,1569,1519,1446,1294,1249,1179,1037,891,830$, 782. HRMS: $\mathrm{m} / \mathrm{z}$ calcd for $\mathrm{C}_{13} \mathrm{H}_{10} \mathrm{ClFO}\left(\mathrm{M}^{+}\right)$: 236.0404, found: 236.0401 .

2,3',6-Trimethoxybiphenyl (3i): Elution with AcOEt / Cyclohexane 4:96 afforded 109 mg (89\% yield) of a yellowish oil. ${ }^{1} \mathrm{H}$ NMR (400 MHz, $\left.\mathrm{CDCl}_{3}\right) \delta(\mathrm{ppm}): 3.76(\mathrm{~s}, 6 \mathrm{H}), 3.84(\mathrm{~s}, 3 \mathrm{H}), 6.68(\mathrm{~d}$, $\left.{ }^{3} J(\mathrm{H}, \mathrm{H})=8.3 \mathrm{~Hz}, 2 \mathrm{H}\right), 6.94(\mathrm{~m}, 3 \mathrm{H}), 7.32(\mathrm{~m}, 2 \mathrm{H})$.

2,2',6-Trimethoxybiphenyl (3j): Elution with AcOEt / Cyclohexane 10:90 afforded 76 mg (62\% yield) of a white solid; mp $138-140{ }^{\circ} \mathrm{C}$ (lit. mp $\left.141{ }^{\circ} \mathrm{C}\right) .{ }^{2 \mathrm{c}}{ }^{1} \mathrm{H} \mathrm{NMR}\left(400 \mathrm{MHz}, \mathrm{CDCl}_{3}\right) \delta$ (ppm): 
$3.74(\mathrm{~s}, 6 \mathrm{H}), 3.77(\mathrm{~s}, 3 \mathrm{H}), 6.67\left(\mathrm{~d},{ }^{3} J(\mathrm{H}, \mathrm{H})=8.3 \mathrm{~Hz}, 2 \mathrm{H}\right), 7.02(\mathrm{~m}, 2 \mathrm{H}), 7.19\left(\mathrm{~d},{ }^{3} J(\mathrm{H}, \mathrm{H})=7.6 \mathrm{~Hz}\right.$, 1H), 7.30 (m, 2H).

2,6-Dimethoxy-4'-methylbiphenyl (3k): Elution with AcOEt / Cyclohexane 2:98 afforded $87 \mathrm{mg}$ (76\% yield) of a white solid; mp 83-85 ${ }^{\circ} \mathrm{C} .{ }^{1} \mathrm{H}$ NMR (400 MHz, $\left.\mathrm{CDCl}_{3}\right) \delta$ (ppm): 2.42 (s, 3H), 3.76 $(\mathrm{s}, 6 \mathrm{H}), 6.67\left(\mathrm{~d},{ }^{3} J(\mathrm{H}, \mathrm{H})=8.3 \mathrm{~Hz}, 2 \mathrm{H}\right), 7.30(\mathrm{~m}, 5 \mathrm{H})$.

4'-Chloro-2,6-dimethoxybiphenyl (31): Elution with AcOEt / Cyclohexane 4:96 afforded $97 \mathrm{mg}$ (78\% yield) of a white solid; mp 118-120 ${ }^{\circ} \mathrm{C} .{ }^{1} \mathrm{H}$ NMR $\left(400 \mathrm{MHz}, \mathrm{CDCl}_{3}\right) \delta(\mathrm{ppm}): 3.75(\mathrm{~s}, 6 \mathrm{H})$, $6.65\left(\mathrm{~d},{ }^{3} \mathrm{~J}(\mathrm{H}, \mathrm{H})=8.3 \mathrm{~Hz}, 2 \mathrm{H}\right), 7.29(\mathrm{~m}, 3 \mathrm{H}), 7.37\left(\mathrm{~d},{ }^{3} \mathrm{~J}(\mathrm{H}, \mathrm{H})=8.3 \mathrm{~Hz}, 2 \mathrm{H}\right) .{ }^{13} \mathrm{C} \mathrm{NMR}(80 \mathrm{MHz}$, $\left.\mathrm{CDCl}_{3}\right) \delta(\mathrm{ppm}): 55.8,104.1,118.2,127.9,129.0,132.3,132.4,132.5,157.5 . \mathrm{IR}\left(\mathrm{CHCl}_{3}\right) \vee\left(\mathrm{cm}^{-1}\right)$ : 3060, 2941, 2908, 1588, 1564, 1472, 1432, 1396, 1268, 1247, 1117, 1102, 730. Anal. Calcd for $\mathrm{C}_{14} \mathrm{H}_{13} \mathrm{ClO}_{2}$ : C 67.61, H 5.27, $\mathrm{Cl}$ 14.26, found: C 67.68, H 5.21, Cl 14.29.

2'-Bromo-2,6-dimethoxybiphenyl (3m): Elution with AcOEt / Cyclohexane 3:97 afforded $85 \mathrm{mg}$ (58\% yield) of a white solid; mp 139-141 ${ }^{\circ} \mathrm{C}$ (lit. mp 141-142 $\left.{ }^{\circ} \mathrm{C}\right) .{ }^{2 \mathrm{a}}{ }^{1} \mathrm{H} \mathrm{NMR}\left(400 \mathrm{MHz}, \mathrm{CDCl}_{3}\right) \delta$ (ppm): $3.77(\mathrm{~s}, 6 \mathrm{H}), 6.68\left(\mathrm{~d},{ }^{3} J(\mathrm{H}, \mathrm{H})=8.3 \mathrm{~Hz}, 2 \mathrm{H}\right), 7.24(\mathrm{~m}, 2 \mathrm{H}), 7.37(\mathrm{~m}, 2 \mathrm{H}), 7.68\left(\mathrm{~d},{ }^{3} J(\mathrm{H}, \mathrm{H})=\right.$ $8.1 \mathrm{~Hz}, 1 \mathrm{H})$.

2,6-Dimethoxybiphenyl (3n): Elution with AcOEt / Cyclohexane 2:98 afforded $89 \mathrm{mg}$ (84\% yield) of a white solid; mp $84-86{ }^{\circ} \mathrm{C}$ (lit. mp 83-85 ${ }^{\circ} \mathrm{C}$ ). ${ }^{2 \mathrm{e}} \mathrm{H}$ NMR $\left(400 \mathrm{MHz}, \mathrm{CDCl}_{3}\right) \delta$ (ppm): 3.77 (s, $6 \mathrm{H}), 6.70\left(\mathrm{~d},{ }^{3} \mathrm{~J}(\mathrm{H}, \mathrm{H})=8.3 \mathrm{~Hz}, 2 \mathrm{H}\right), 7.36(\mathrm{~m}, 6 \mathrm{H})$.

2,6-Dimethoxy-3'-(trifluoromethyl)biphenyl (3o): Elution with AcOEt / Cyclohexane 5:95 afforded $109 \mathrm{mg}$ (77\% yield) of a white solid; mp 77-79 ${ }^{\circ} \mathrm{C} .{ }^{1} \mathrm{H} \mathrm{NMR}\left(400 \mathrm{MHz}, \mathrm{CDCl}_{3}\right) \delta$ (ppm): $3.76(\mathrm{~s}, 6 \mathrm{H}), 6.67\left(\mathrm{~d},{ }^{3} J(\mathrm{H}, \mathrm{H})=8.3 \mathrm{~Hz}, 2 \mathrm{H}\right), 7.32\left(\mathrm{t},{ }^{3} J(\mathrm{H}, \mathrm{H})=8.3 \mathrm{~Hz}, 1 \mathrm{H}\right), 7.58(\mathrm{~m}, 4 \mathrm{H})$.

2,6-Dimethoxy-4'-acetylbiphenyl (3p): Elution with AcOEt / Cyclohexane 15:85 afforded $89 \mathrm{mg}$ (70\% yield) of a white solid; mp 137-139 ${ }^{\circ} \mathrm{C} .{ }^{1} \mathrm{H}$ NMR (400 MHz, $\left.\mathrm{CDCl}_{3}\right) \delta$ (ppm): 2.64 (s, 3H), $3.75(\mathrm{~s}, 6 \mathrm{H}), 6.67\left(\mathrm{~d},{ }^{3} J(\mathrm{H}, \mathrm{H})=8.3 \mathrm{~Hz}, 2 \mathrm{H}\right), 7.32\left(\mathrm{t},{ }^{3} J(\mathrm{H}, \mathrm{H})=8.3 \mathrm{~Hz}, 1 \mathrm{H}\right), 7.47\left(\mathrm{~d},{ }^{3} J(\mathrm{H}, \mathrm{H})=8.3\right.$ $\mathrm{Hz}, 2 \mathrm{H}), 8.01\left(\mathrm{~d},{ }^{3} \mathrm{~J}(\mathrm{H}, \mathrm{H})=8.3 \mathrm{~Hz}, 2 \mathrm{H}\right)$.

2,6-Dimethoxy-2'-nitrobiphenyl (3q): Elution with AcOEt / Cyclohexane 10:90 afforded 92 mg (71\% yield) of a yellow oil. ${ }^{1} \mathrm{H}$ NMR $\left(400 \mathrm{MHz}, \mathrm{CDCl}_{3}\right) \delta(\mathrm{ppm}): 3.72(\mathrm{~s}, 6 \mathrm{H}), 6.65\left(\mathrm{~d},{ }^{3} J(\mathrm{H}, \mathrm{H})=\right.$ $8.6 \mathrm{~Hz}, 2 \mathrm{H}), 7.33\left(\mathrm{t},{ }^{3} J(\mathrm{H}, \mathrm{H})=8.6 \mathrm{~Hz}, 1 \mathrm{H}\right), 7.48(\mathrm{~m}, 2 \mathrm{H}), 7.64\left(\mathrm{t},{ }^{3} J(\mathrm{H}, \mathrm{H})=8.1 \mathrm{~Hz}, 1 \mathrm{H}\right), 7.99(\mathrm{~d}$, $\left.{ }^{3} J(\mathrm{H}, \mathrm{H})=8.1 \mathrm{~Hz}, 1 \mathrm{H}\right) \cdot{ }^{13} \mathrm{C} \mathrm{NMR}\left(80 \mathrm{MHz}, \mathrm{CDCl}_{3}\right) \delta(\mathrm{ppm}): 55.8,104.2,115.2,123.9,127.8$, 129.2, 129.7, 132.1, 133.7, 149.8, 156.9. IR $\left(\mathrm{CHCl}_{3}\right) \vee\left(\mathrm{cm}^{-1}\right): 3002,2928,2362,1612,1591,1525$, 1474, 1432, 1355, 1253, 1110, 782. HRMS: m/z calcd for $\mathrm{C}_{14} \mathrm{H}_{13} \mathrm{NO}_{4}\left(\mathrm{M}^{+}\right)$: 259.0845, found: 259.0838 .

Ethyl 2',6'-Dimethoxybiphenyl-2-carboxylate (3r): Elution with AcOEt / Cyclohexane 5:95 afforded $84 \mathrm{mg}$ (59\% yield) of a yellowish oil. ${ }^{1} \mathrm{H}$ NMR (400 $\left.\mathrm{MHz}, \mathrm{CDCl}_{3}\right) \delta(\mathrm{ppm}): 1.01(\mathrm{t}$, 
$\left.{ }^{3} J(\mathrm{H}, \mathrm{H})=7.1 \mathrm{~Hz}, 3 \mathrm{H}\right), 3.70(\mathrm{~s}, 6 \mathrm{H}), 4.07\left(\mathrm{q},{ }^{3} J(\mathrm{H}, \mathrm{H})=7.1 \mathrm{~Hz}, 2 \mathrm{H}\right), 6.63\left(\mathrm{~d},{ }^{3} J(\mathrm{H}, \mathrm{H})=8.3 \mathrm{~Hz}, 2 \mathrm{H}\right)$, $7.31(\mathrm{~m}, 2 \mathrm{H}), 7.40\left(\mathrm{t},{ }^{3} J(\mathrm{H}, \mathrm{H})=8.3 \mathrm{~Hz}, 1 \mathrm{H}\right), 7.54\left(\mathrm{~d},{ }^{3} J(\mathrm{H}, \mathrm{H})=7.8 \mathrm{~Hz}, 1 \mathrm{H}\right), 7.96\left(\mathrm{~d},{ }^{3} J(\mathrm{H}, \mathrm{H})=7.8\right.$ $\mathrm{Hz}, 1 \mathrm{H}) .{ }^{13} \mathrm{C} \mathrm{NMR}\left(80 \mathrm{MHz}, \mathrm{CDCl}_{3}\right) \delta(\mathrm{ppm}): 13.7,55.8,60.3,104.0,119.3,126.9,128.6,129.7$, 131.1, 132.0, 132.3, 134.8, 157.1, 167.9. IR $\left(\mathrm{CHCl}_{3}\right) \vee\left(\mathrm{cm}^{-1}\right): 3061,2958,1724,1591,1471,1432$, 1365, 1289, 1250, 1110, 1045, 785. HRMS: m/z calcd for $\mathrm{C}_{17} \mathrm{H}_{18} \mathrm{O}_{4}\left(\mathrm{M}^{+}\right)$: 286.1205, found: 286.1200 .

2-(4-Methoxyphenyl)-3-methylbenzofurane (3s): Elution with AcOEt / Cyclohexane 1:99 afforded $77 \mathrm{mg}$ (65\% yield) of a yellow solid; mp 86-88 ${ }^{\circ} \mathrm{C}$ (lit. $\left.\mathrm{mp} 87{ }^{\circ} \mathrm{C}\right) .{ }^{2 \mathrm{~g}}{ }^{1} \mathrm{H} \mathrm{NMR}(400 \mathrm{MHz}$, $\left.\mathrm{CDCl}_{3}\right) \delta(\mathrm{ppm}): 2.40(\mathrm{~s}, 3 \mathrm{H}), 3.82(\mathrm{~s}, 3 \mathrm{H}), 6.96\left(\mathrm{~d},{ }^{3} J(\mathrm{H}, \mathrm{H})=8.8 \mathrm{~Hz}, 2 \mathrm{H}\right), 7.22(\mathrm{~m}, 2 \mathrm{H}), 7.44(\mathrm{~m}$, $2 \mathrm{H}), 7.69\left(\mathrm{~d},{ }^{3} \mathrm{~J}(\mathrm{H}, \mathrm{H})=8.8 \mathrm{~Hz}, 2 \mathrm{H}\right)$.

1-(2,6-Dimethoxyphenyl)naphthalene (3t): Elution with AcOEt / Cyclohexane 3:97 afforded 96 mg (73\% yield) of a yellowish solid; mp 144-146 ${ }^{\circ} \mathrm{C}$ (lit. mp $\left.147{ }^{\circ} \mathrm{C}\right) .{ }^{2 \mathrm{~h}}{ }^{1} \mathrm{H}$ NMR (400 MHz, $\left.\mathrm{CDCl}_{3}\right) \delta(\mathrm{ppm}): 3.66(\mathrm{~s}, 6 \mathrm{H}), 6.74\left(\mathrm{~d},{ }^{3} J(\mathrm{H}, \mathrm{H})=8.0 \mathrm{~Hz}, 2 \mathrm{H}\right), 7.42(\mathrm{~m}, 5 \mathrm{H}), 7.56\left(\mathrm{t},{ }^{3} J(\mathrm{H}, \mathrm{H})=8.0\right.$ $\mathrm{Hz}, 1 \mathrm{H}), 7.88(\mathrm{~m}, 2 \mathrm{H})$. 


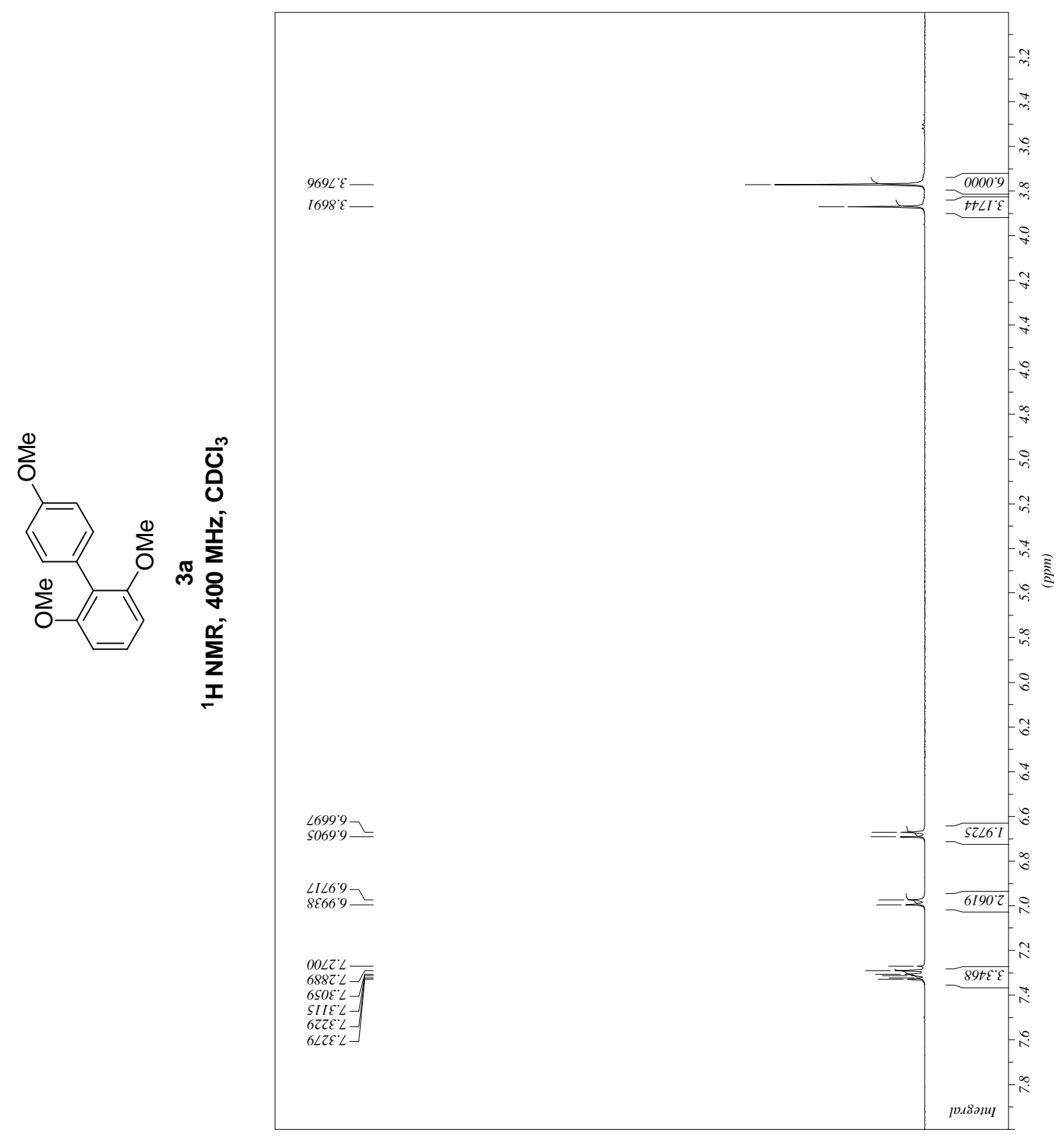




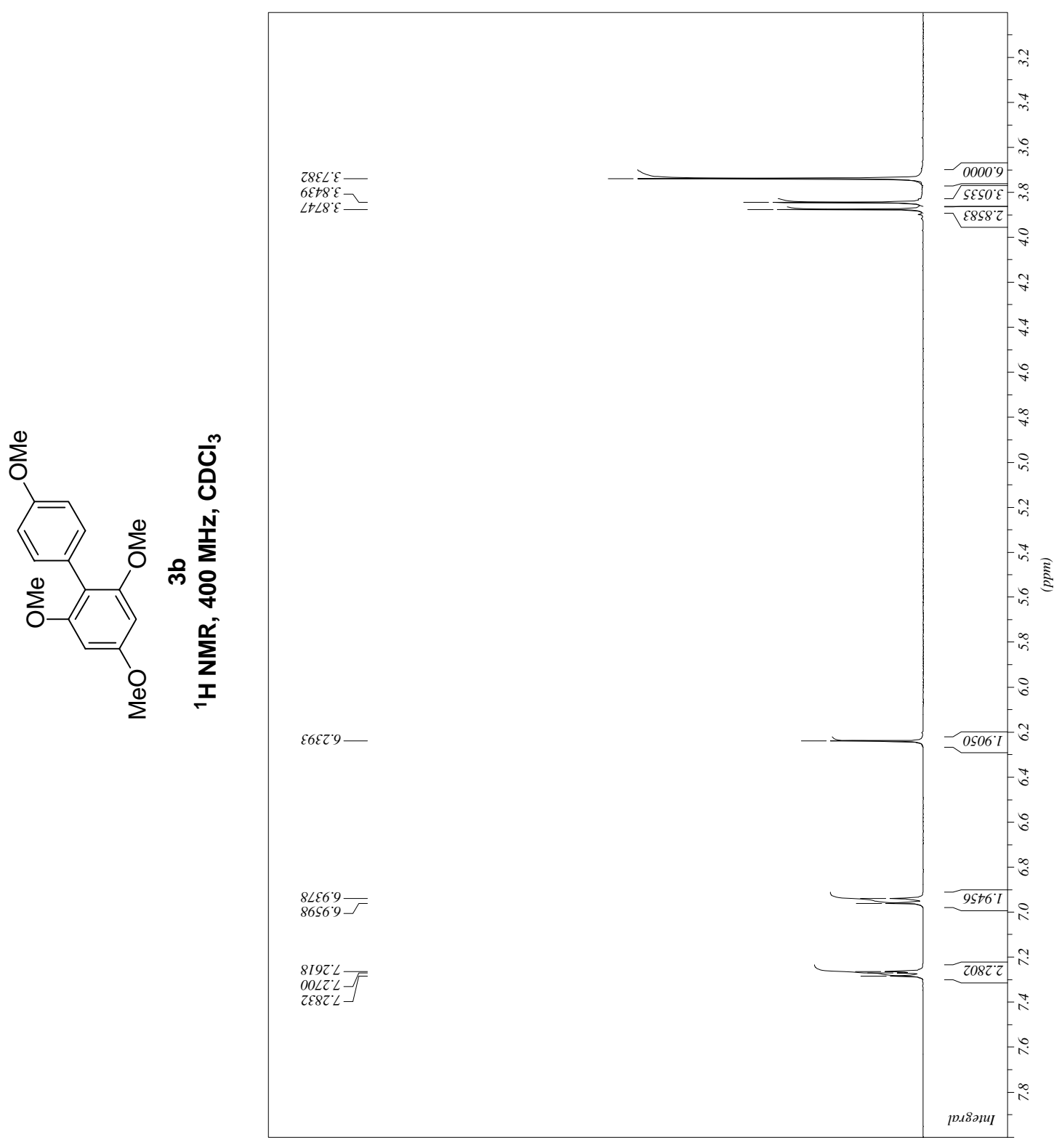




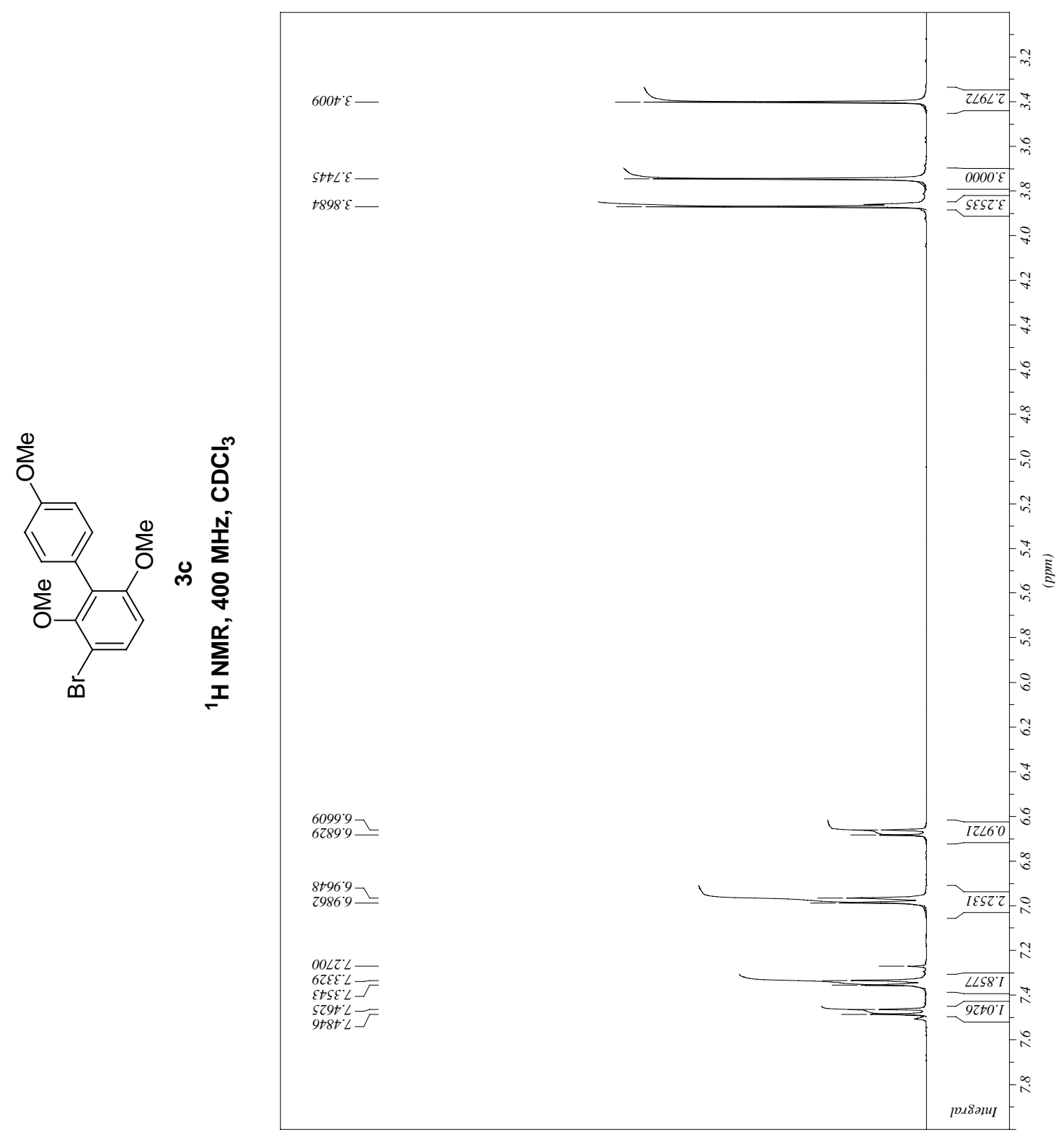




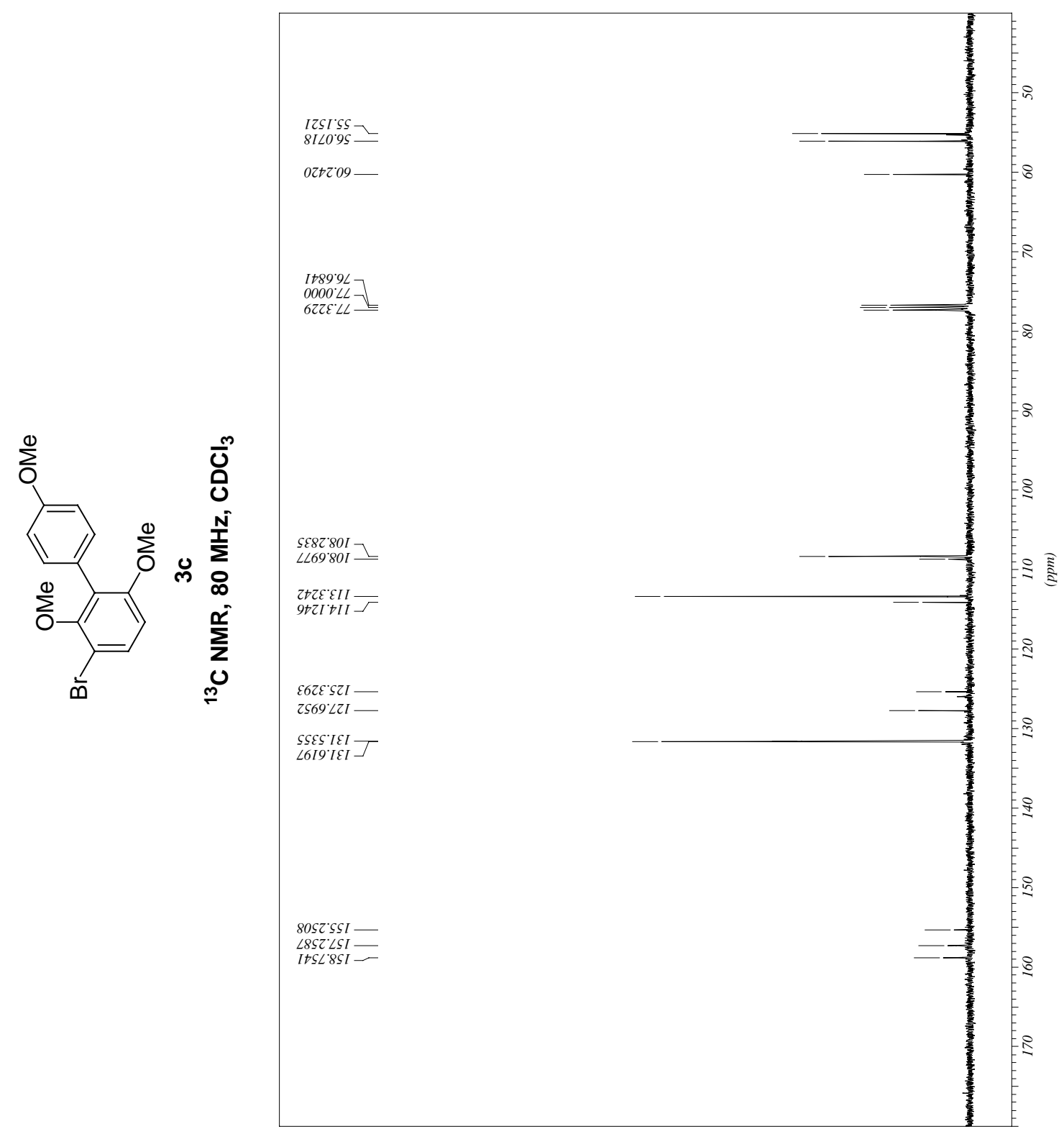




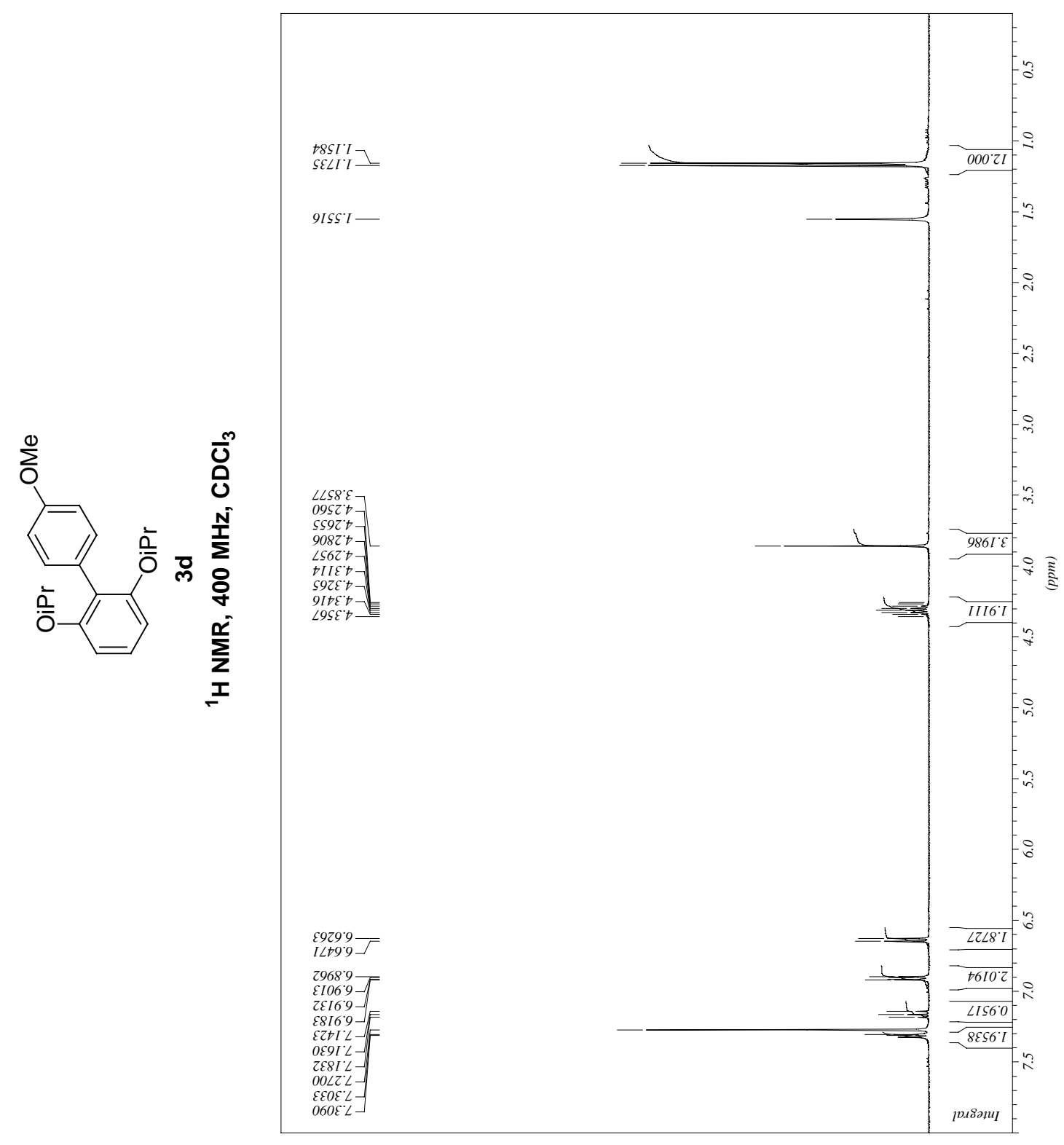




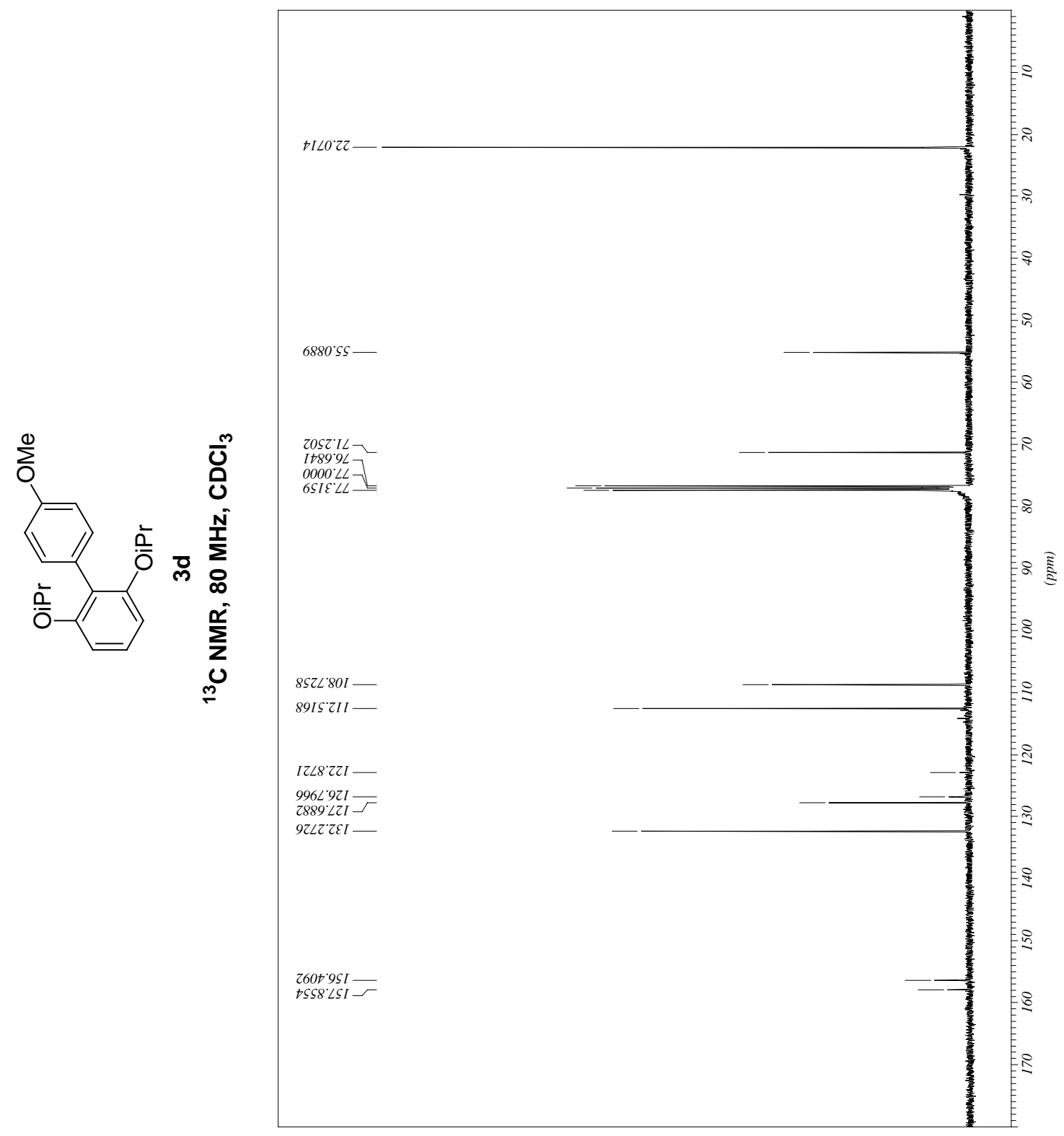




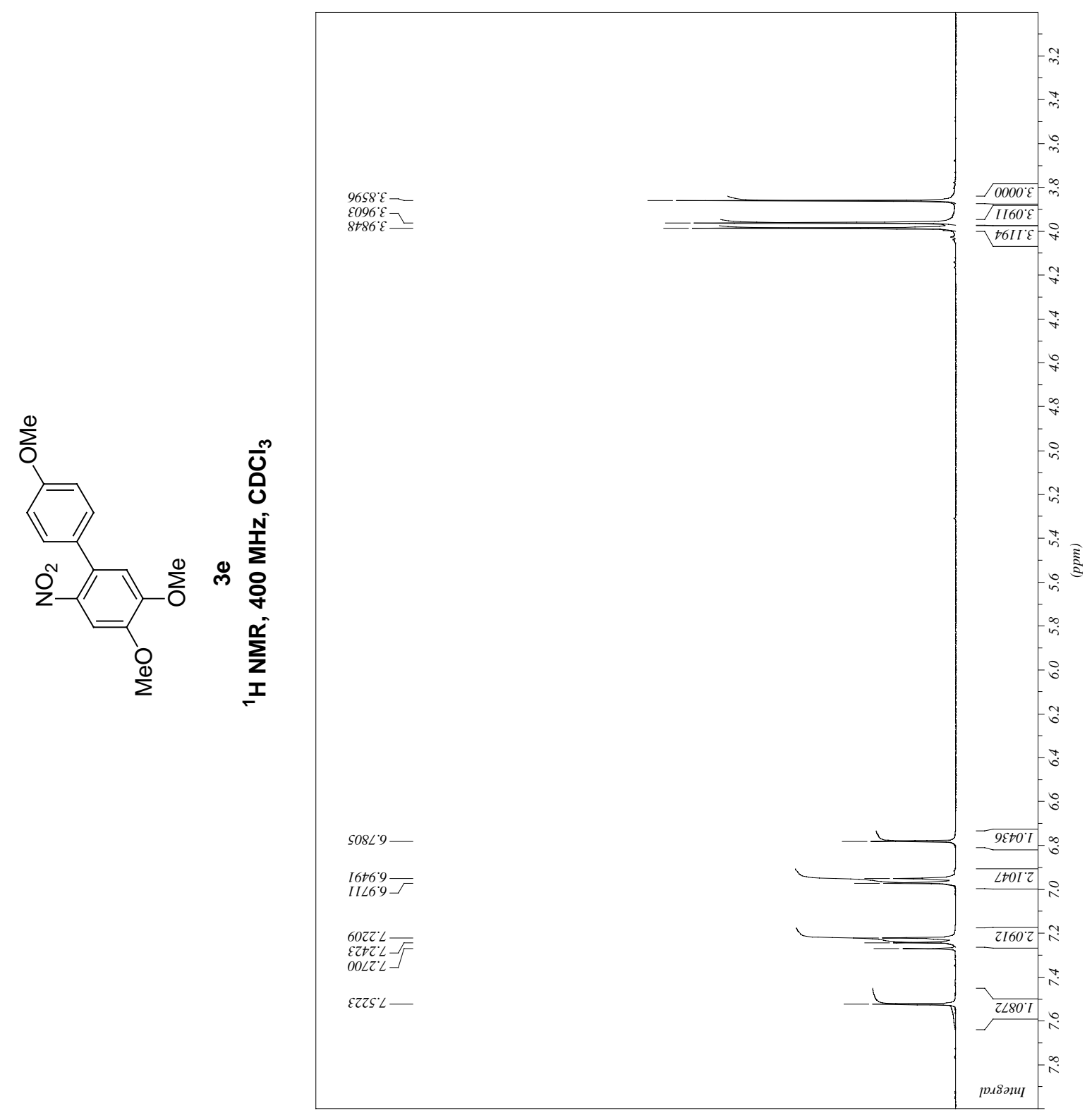




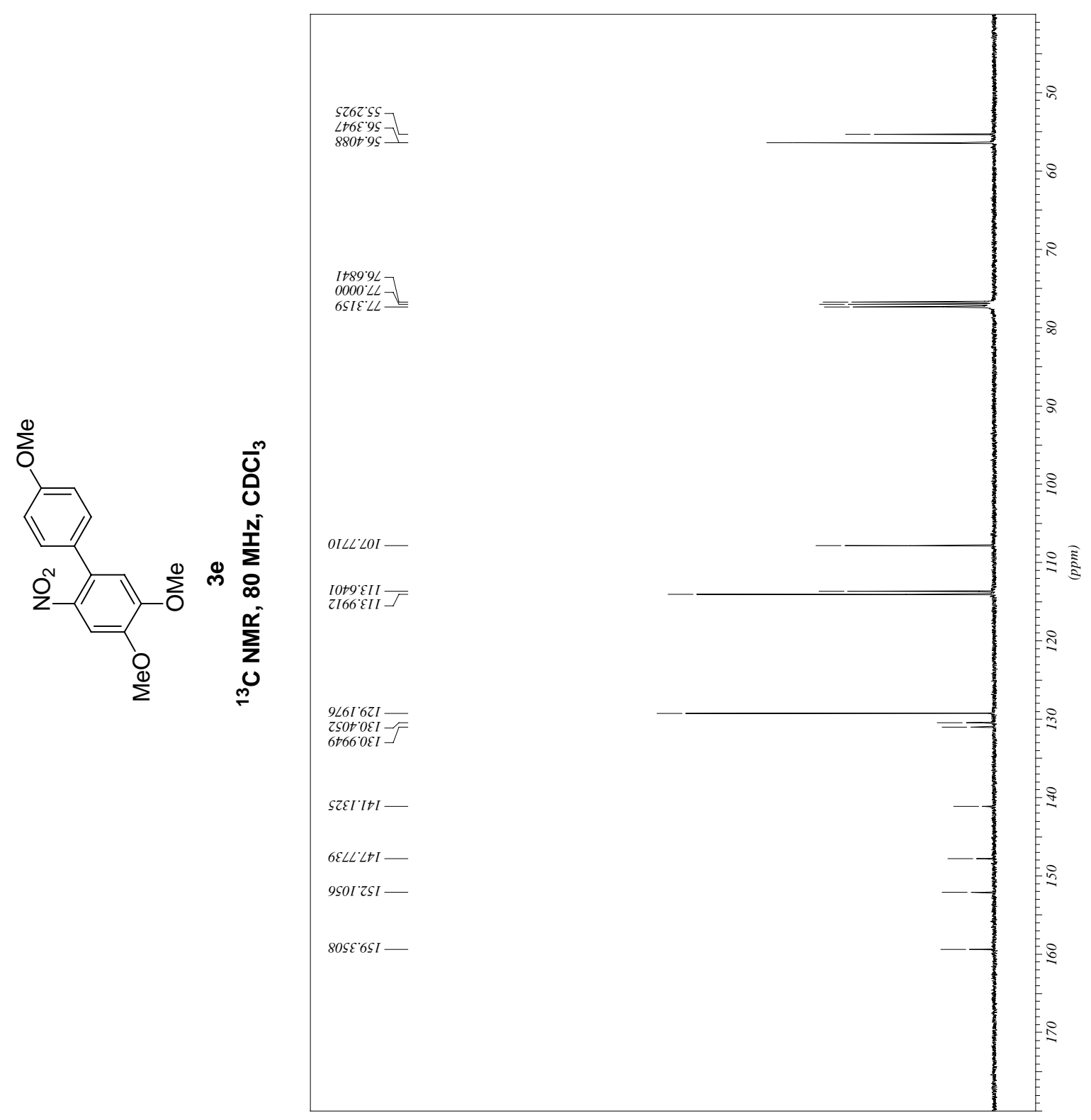




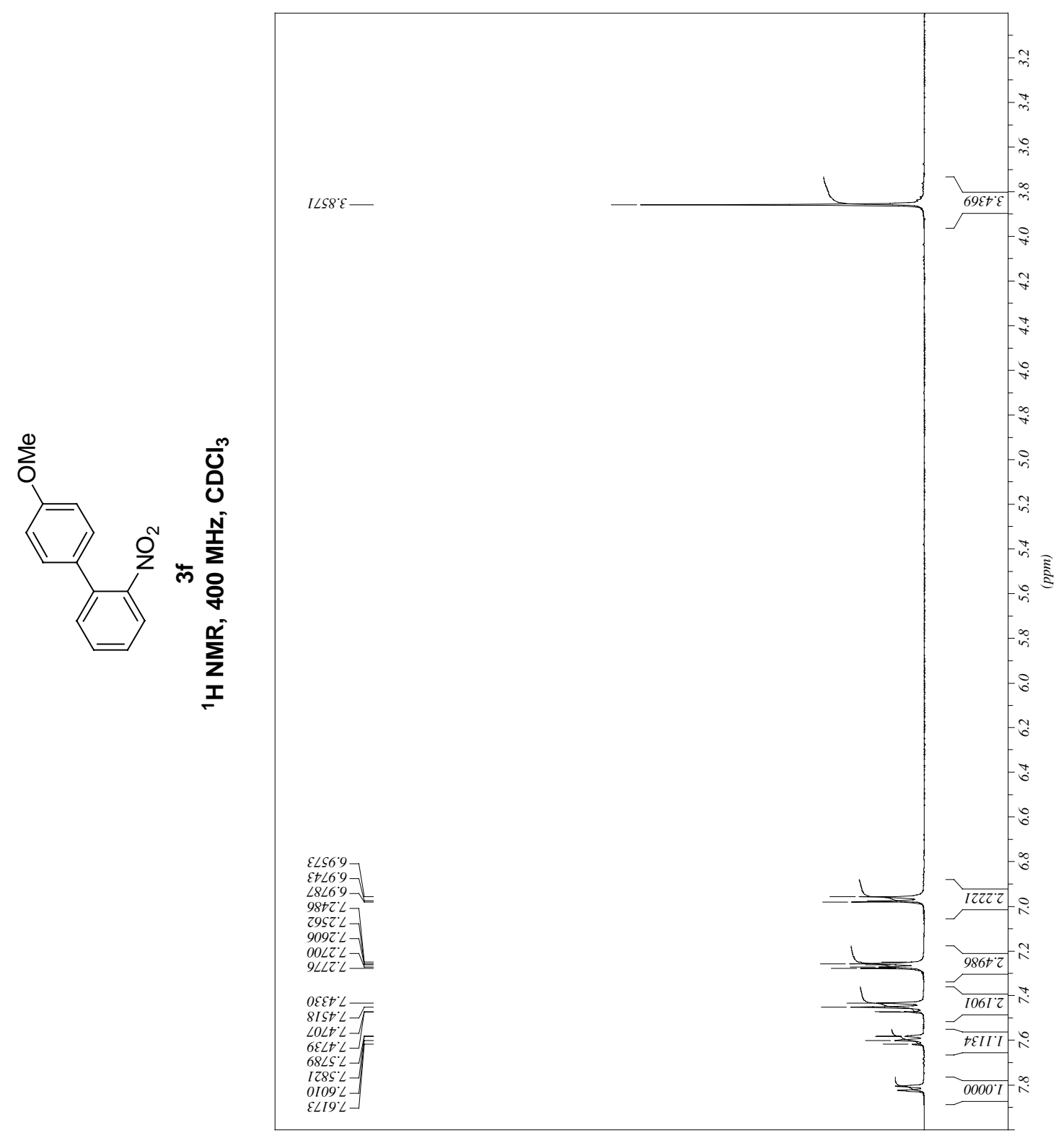




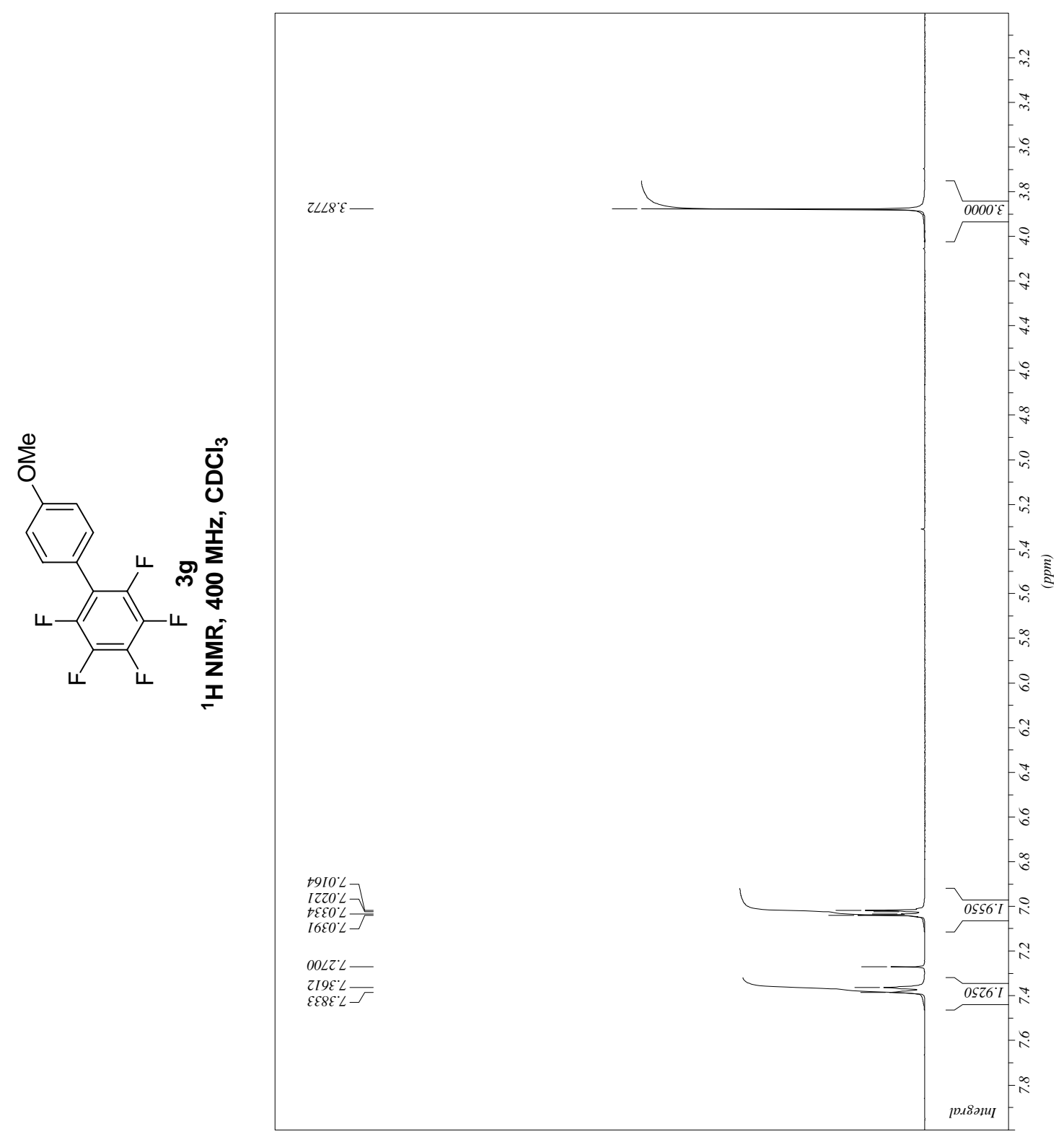




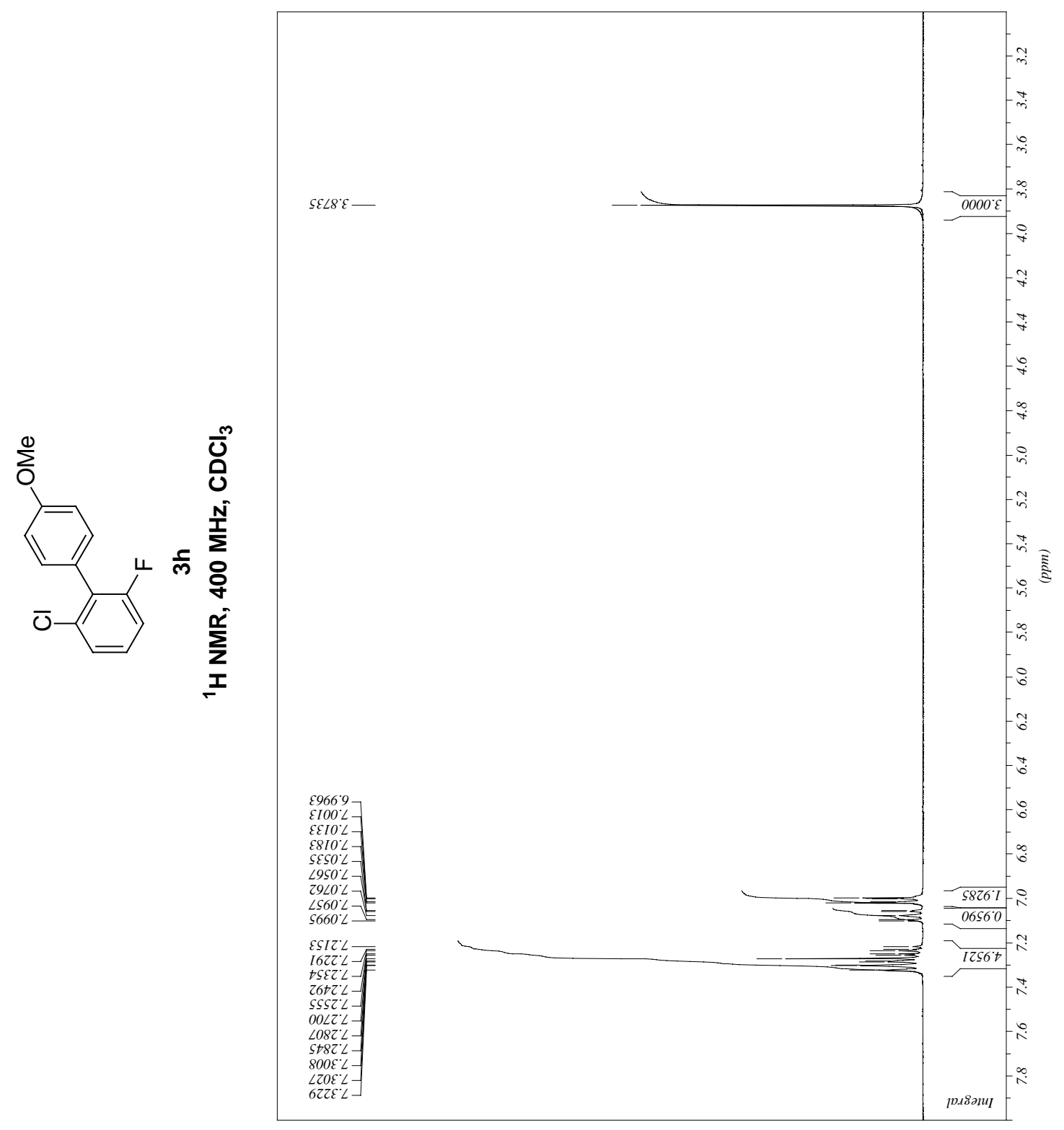




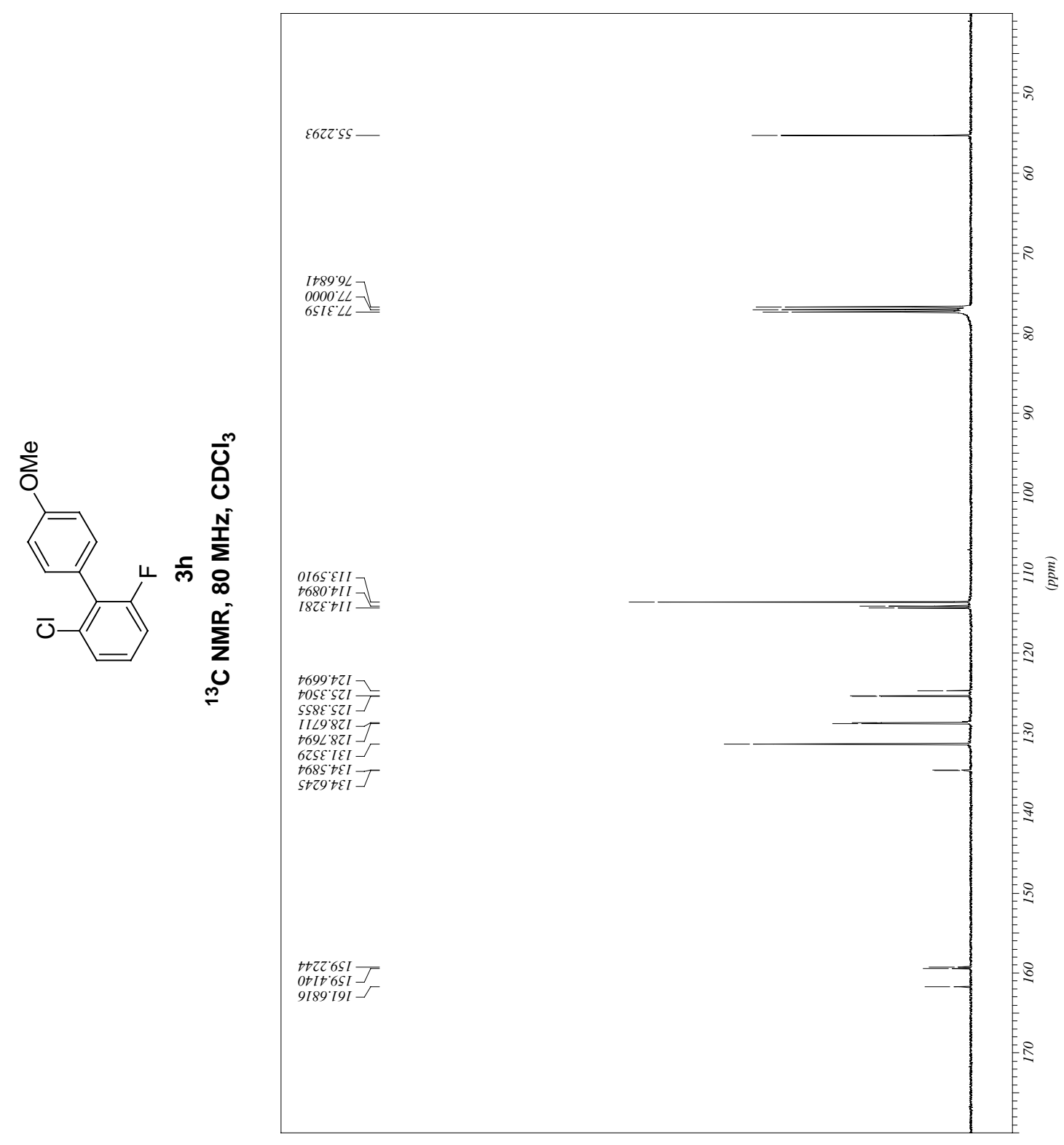




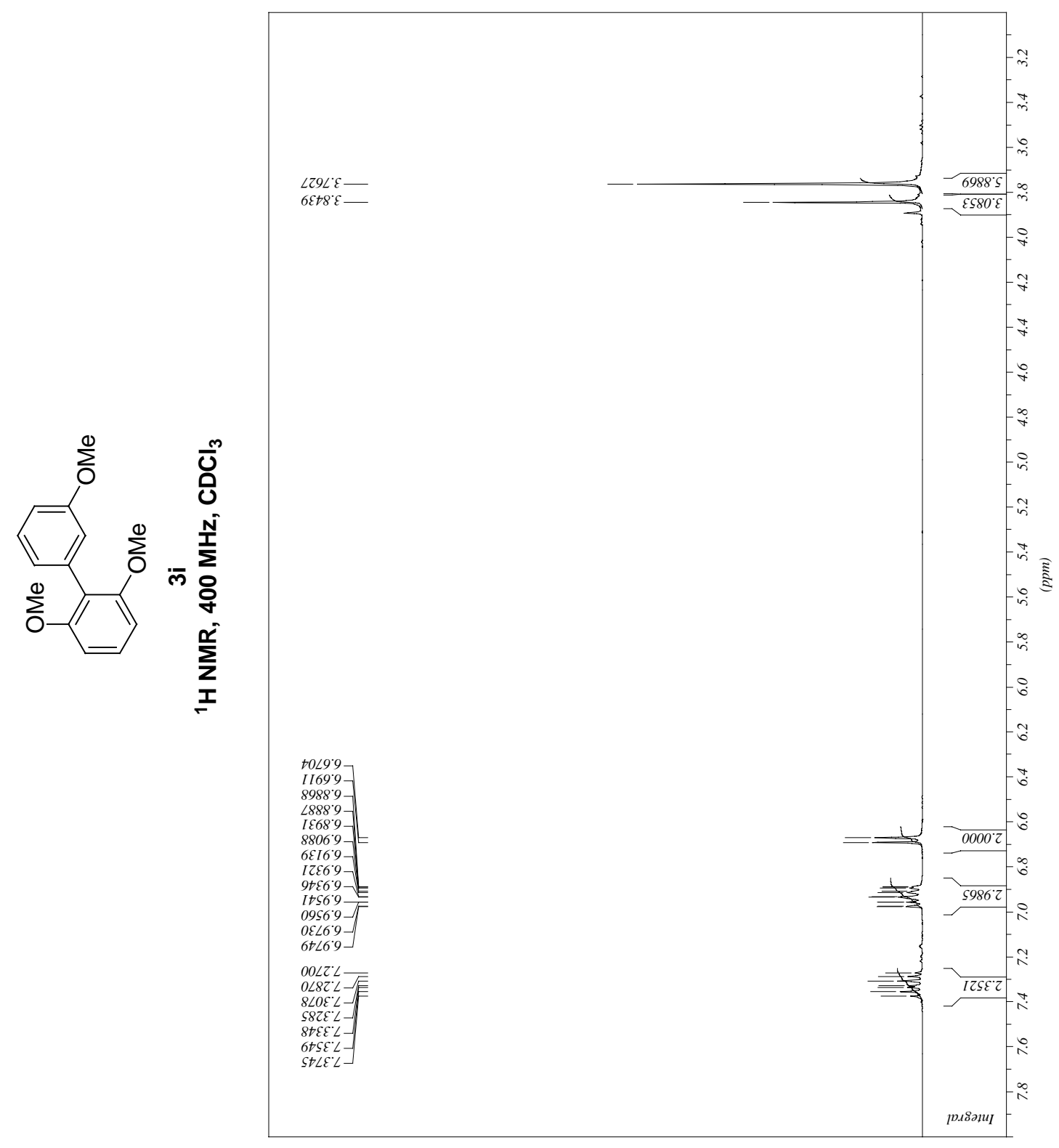




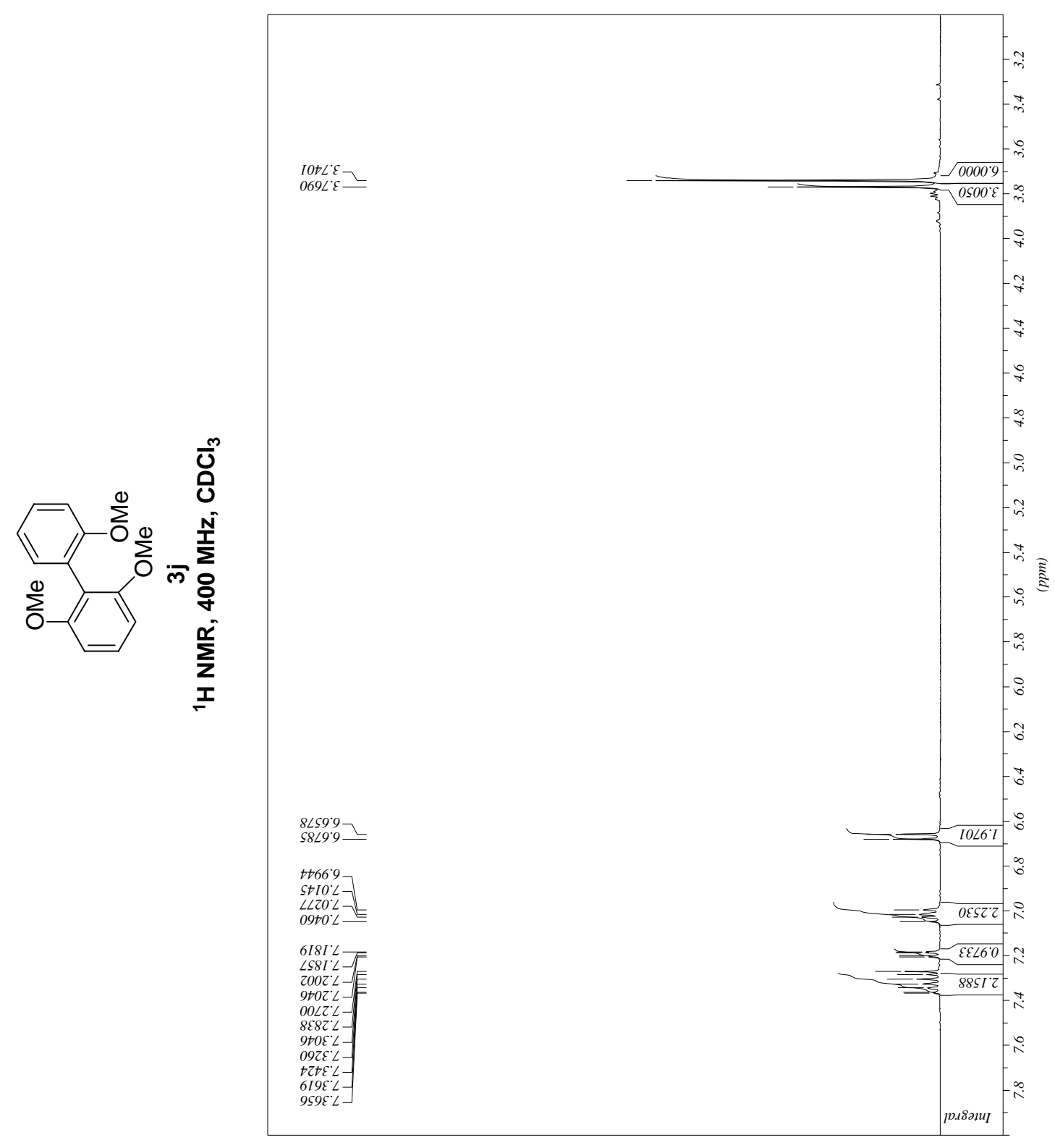




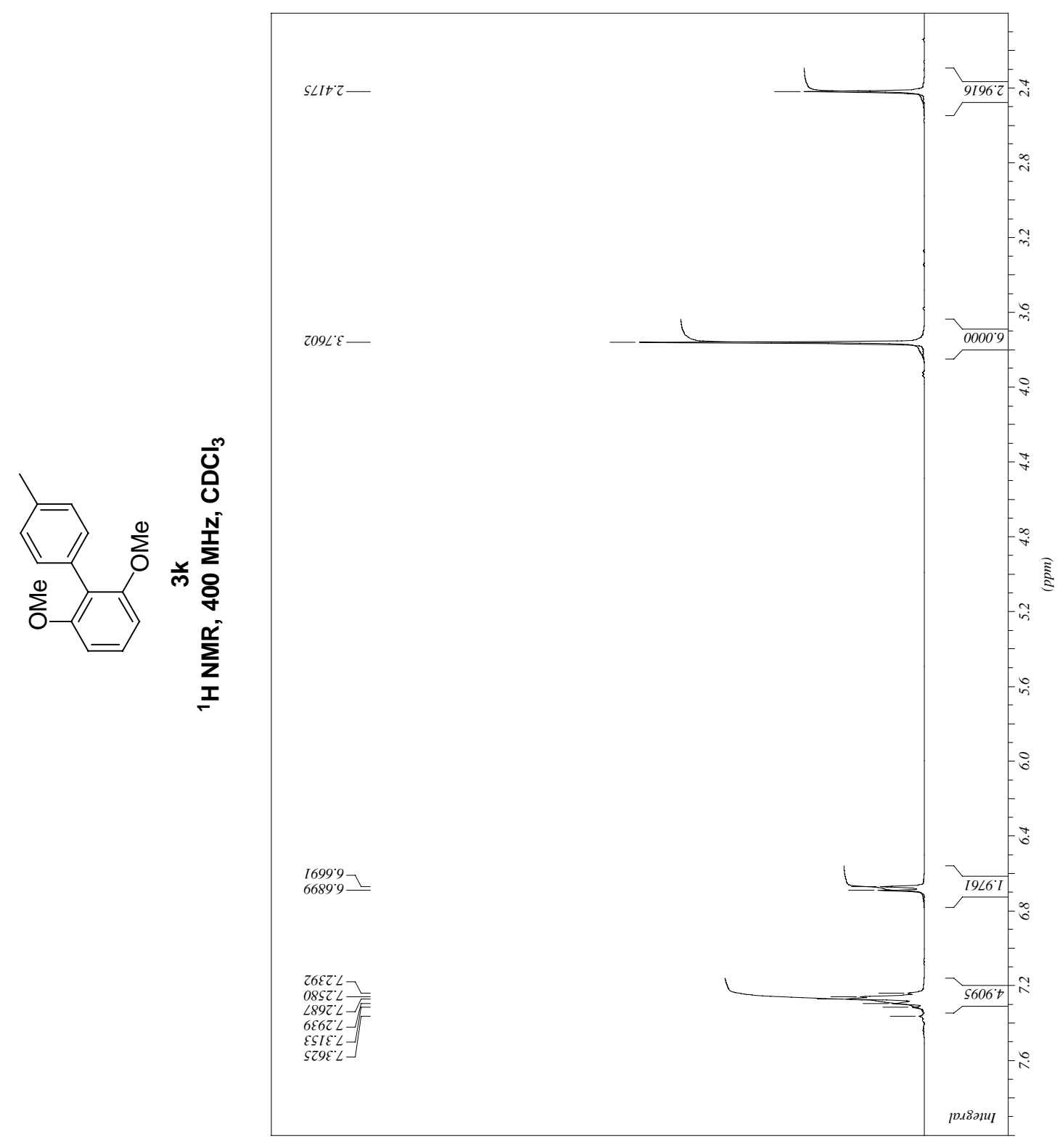




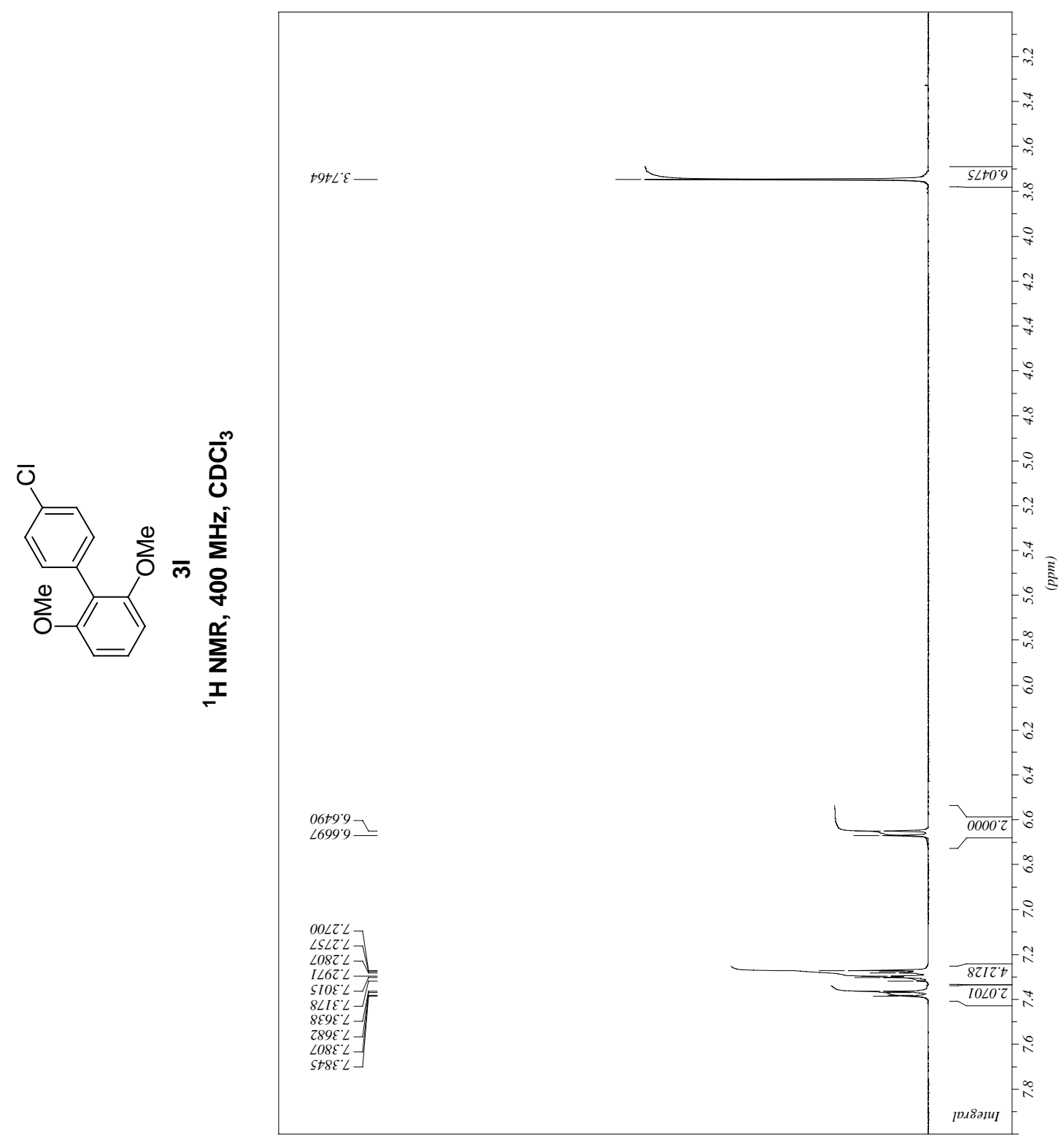




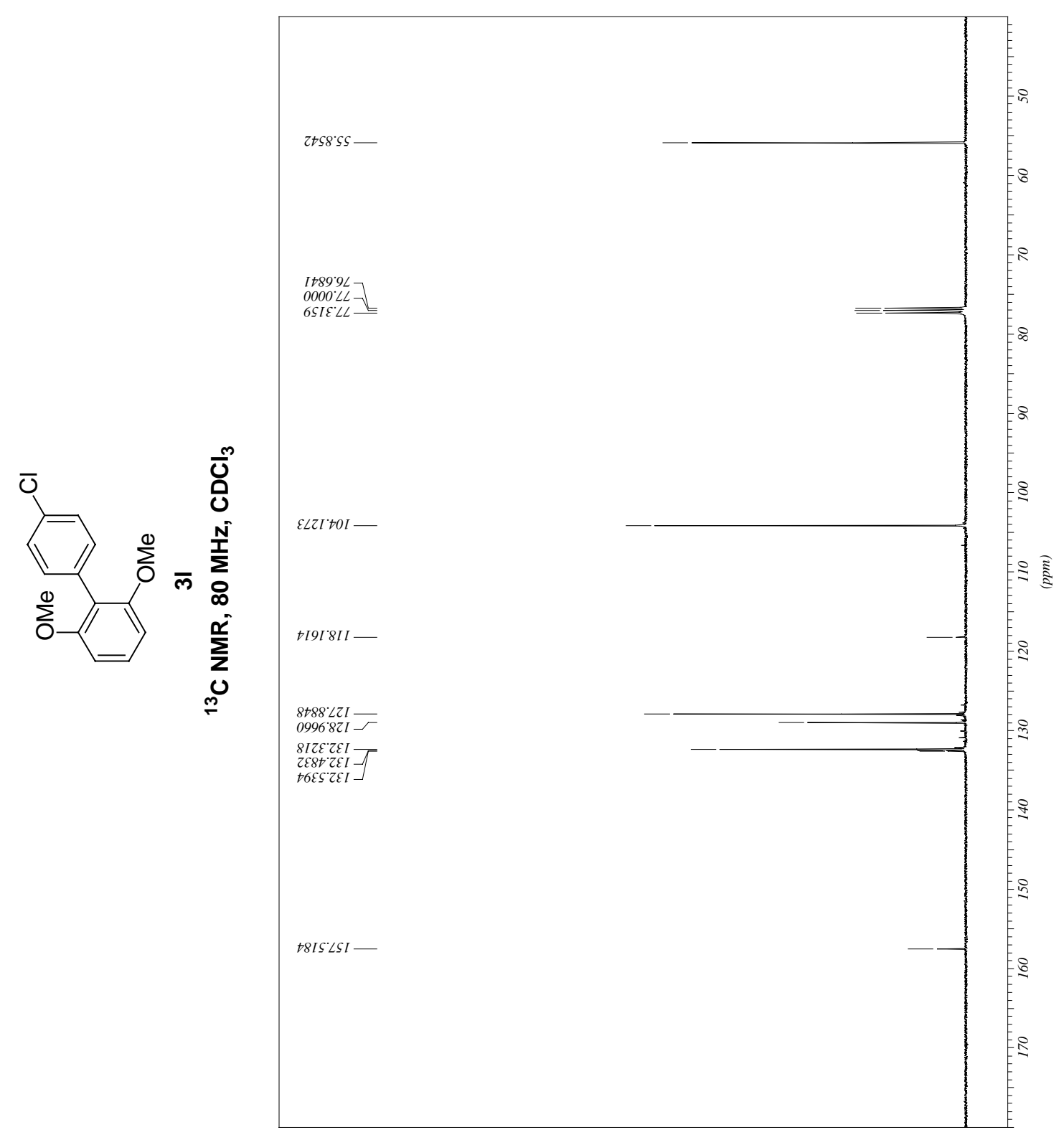




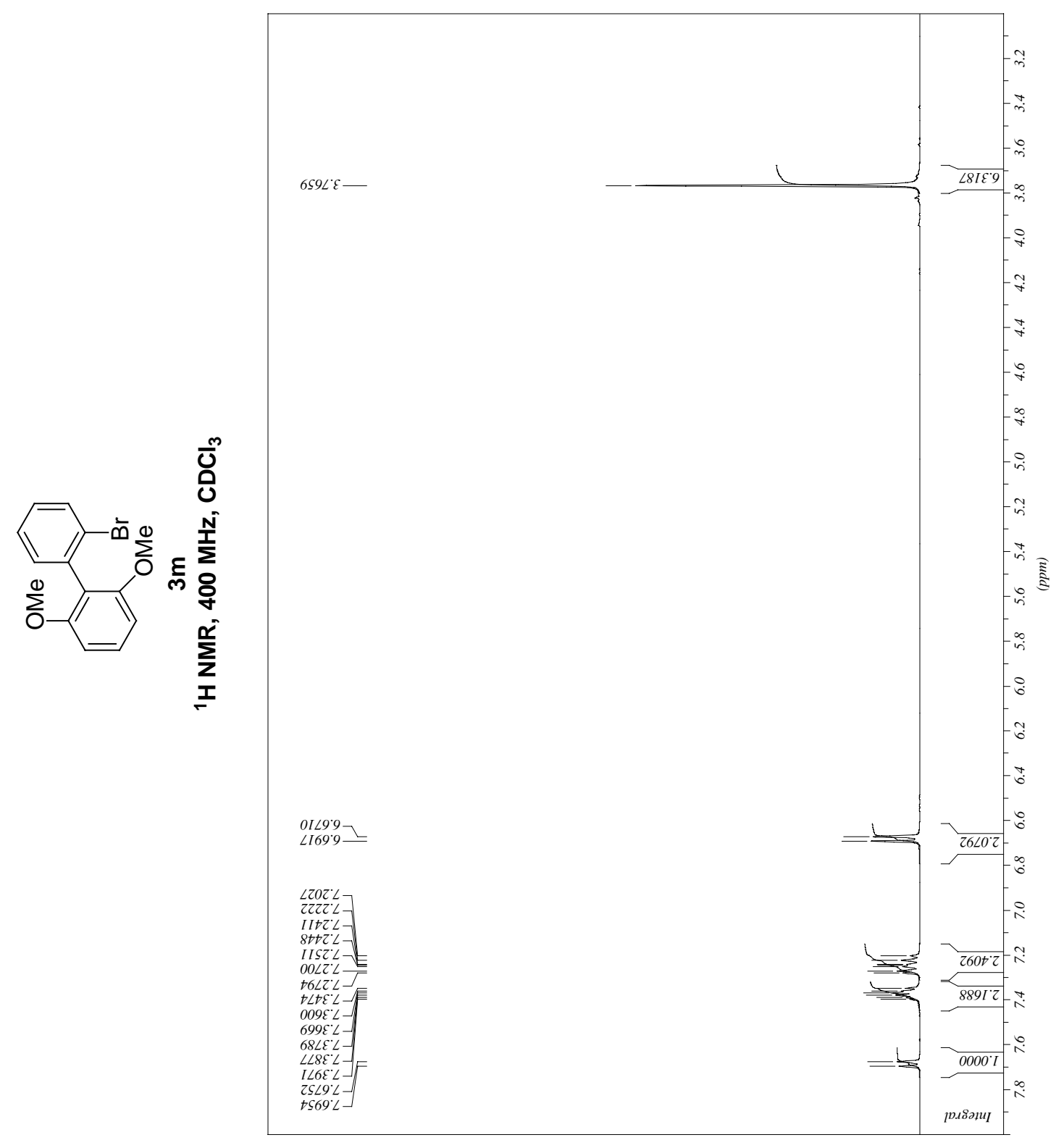




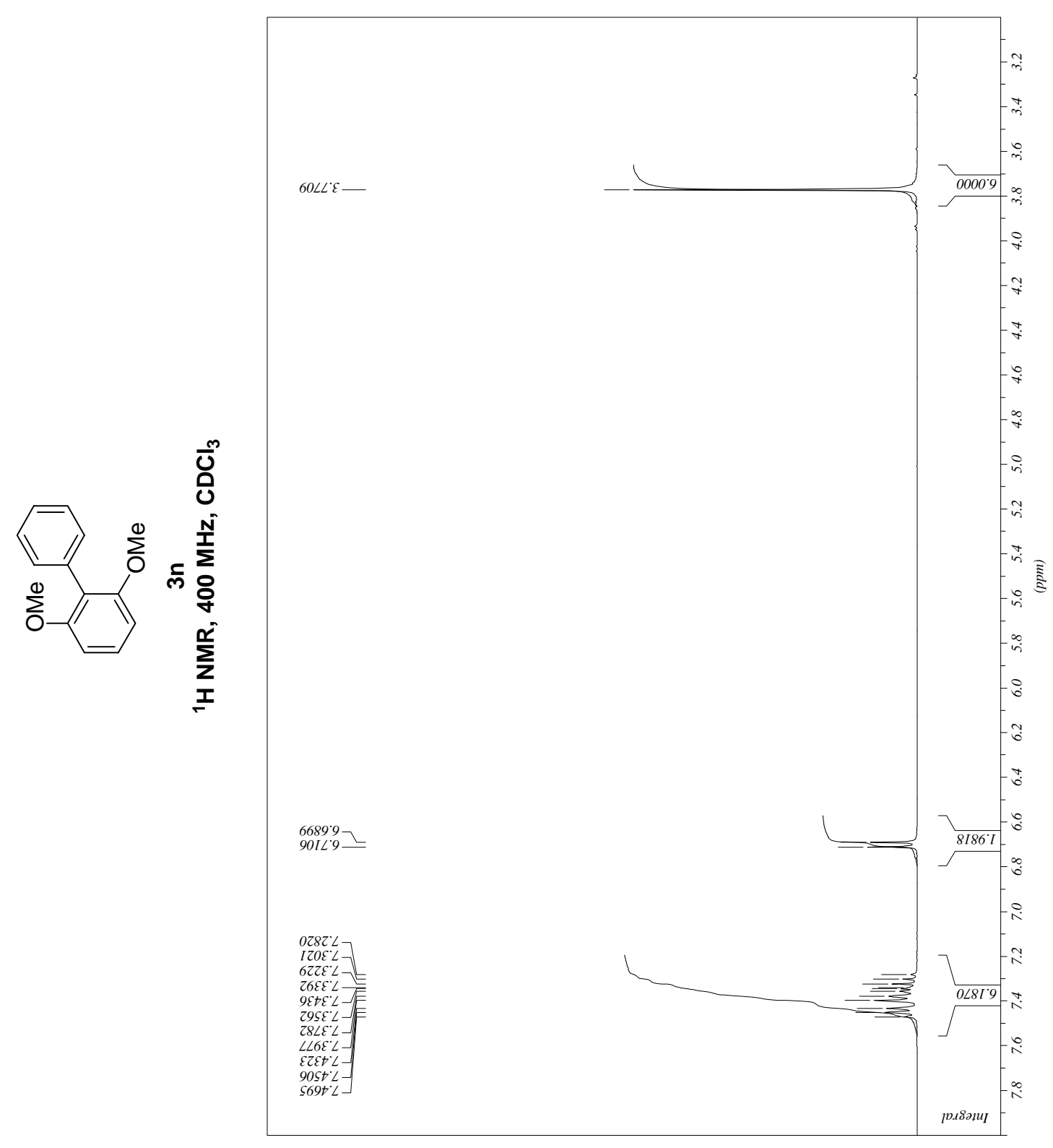




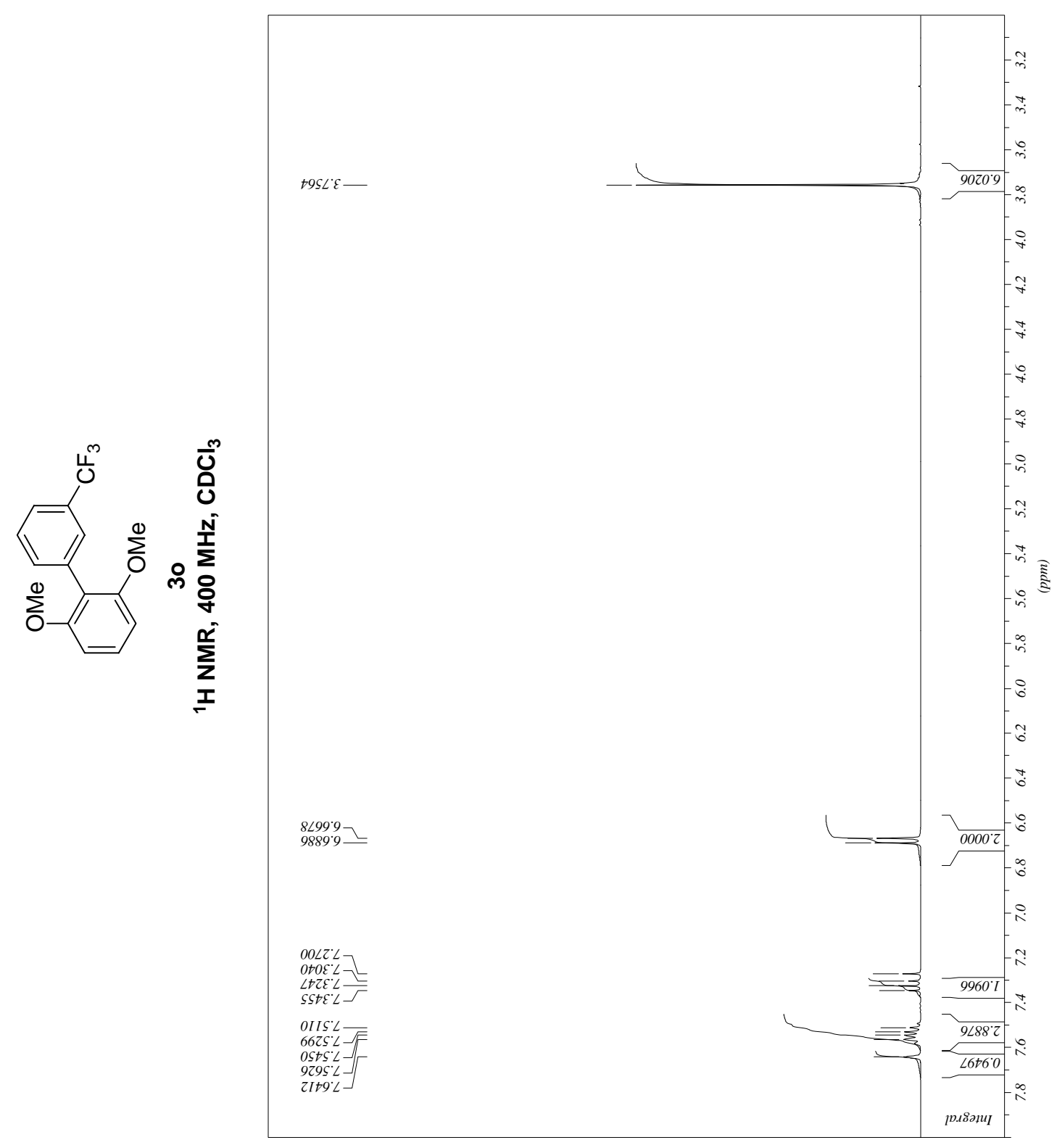




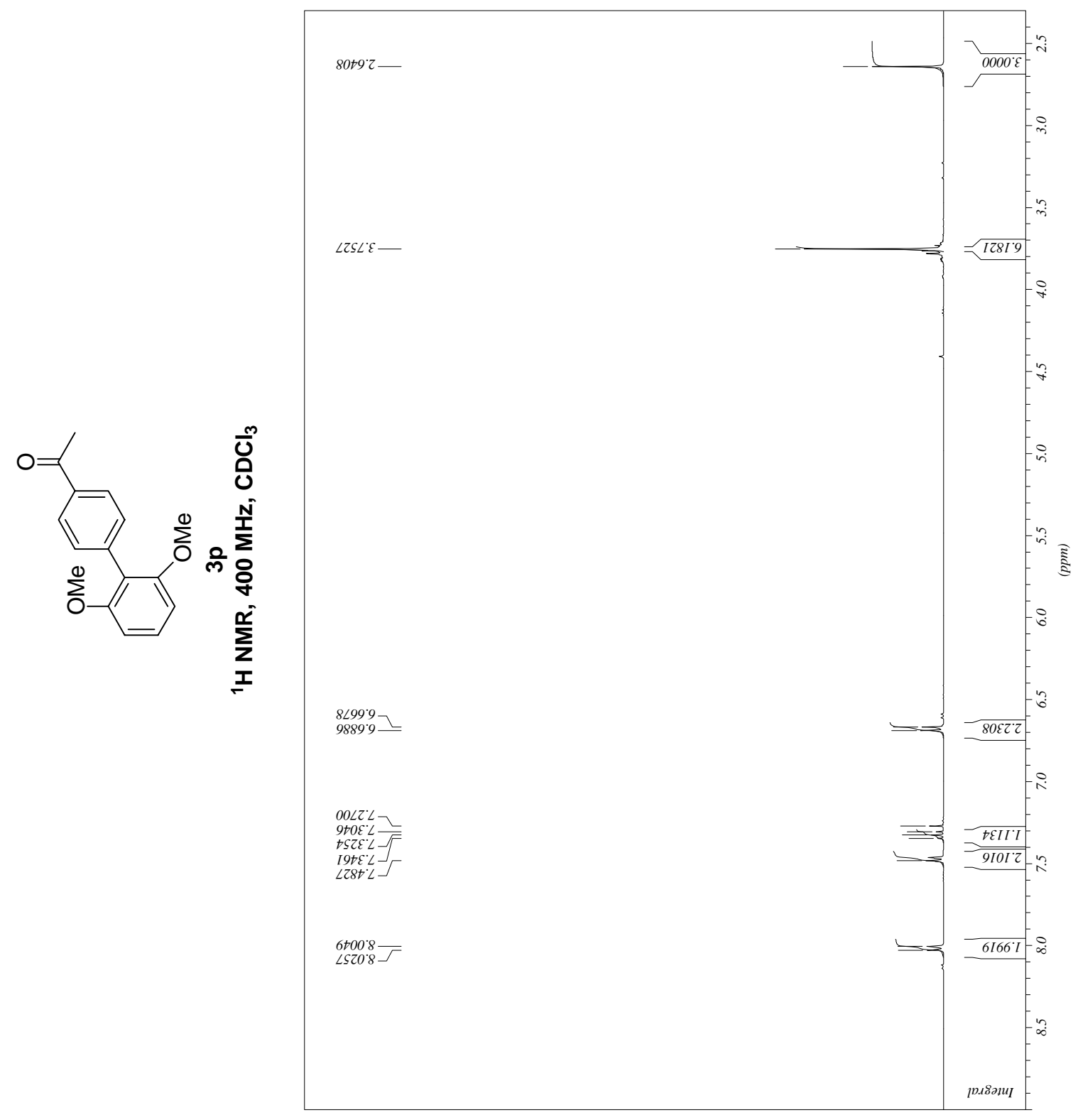




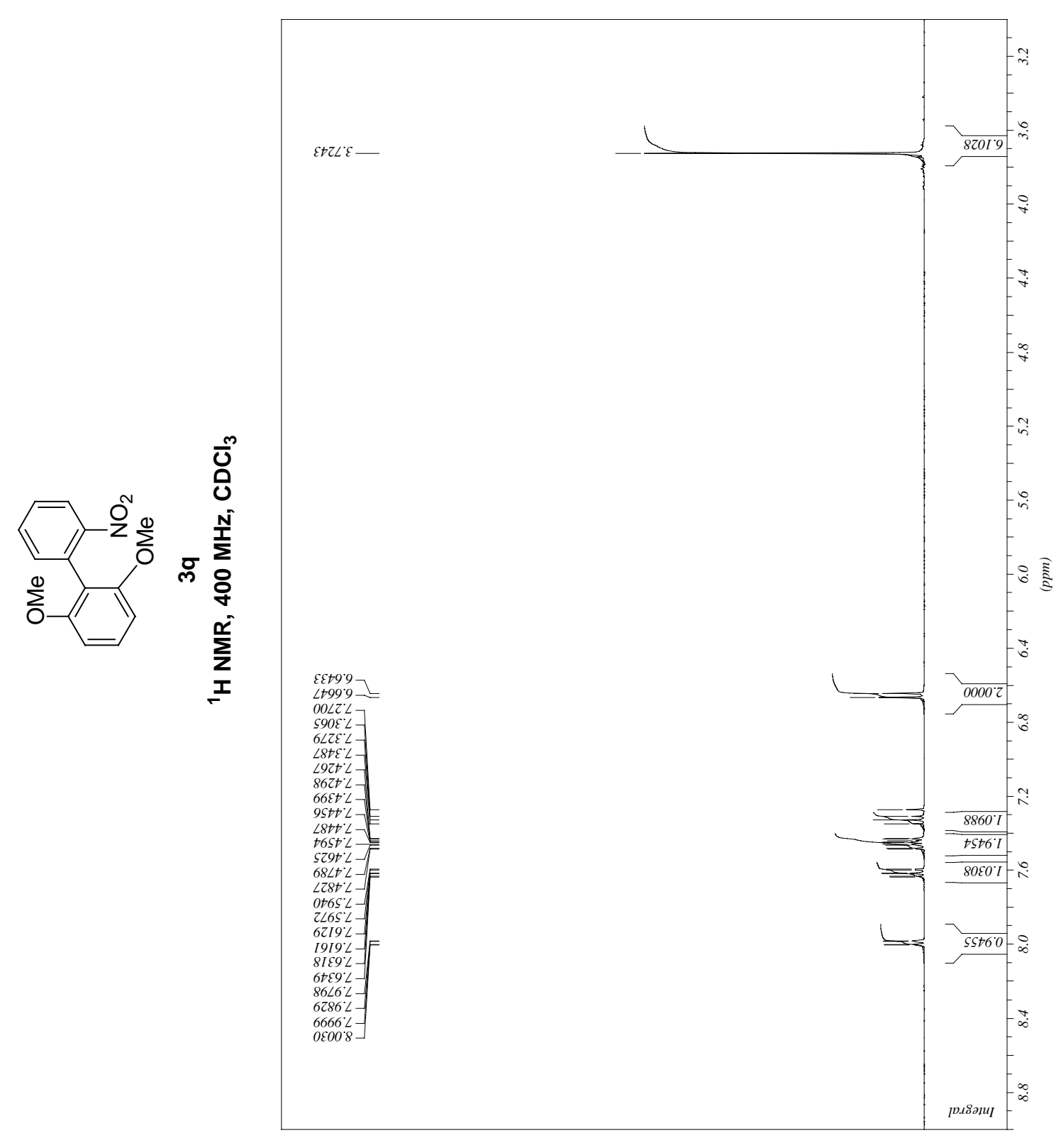




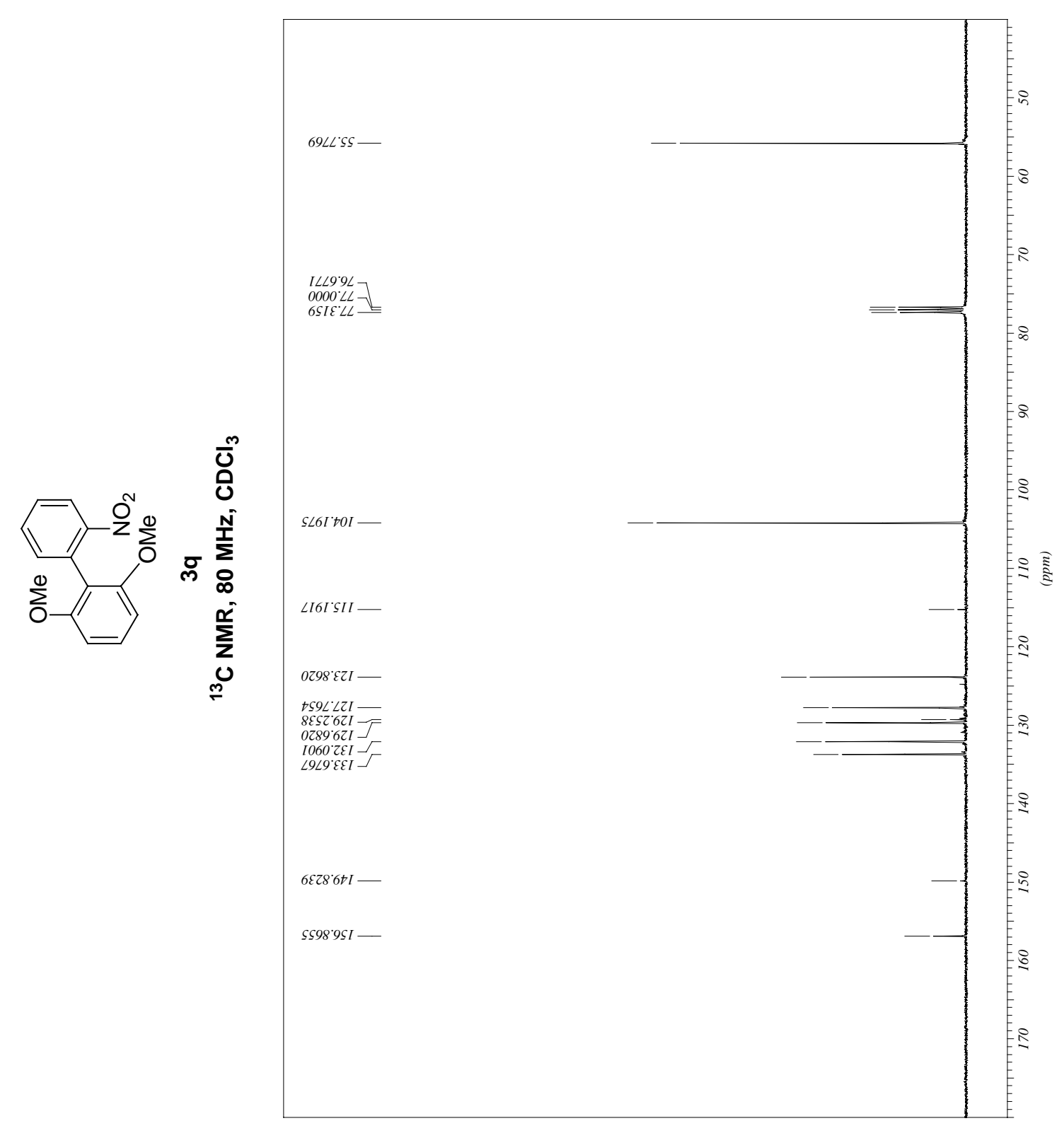




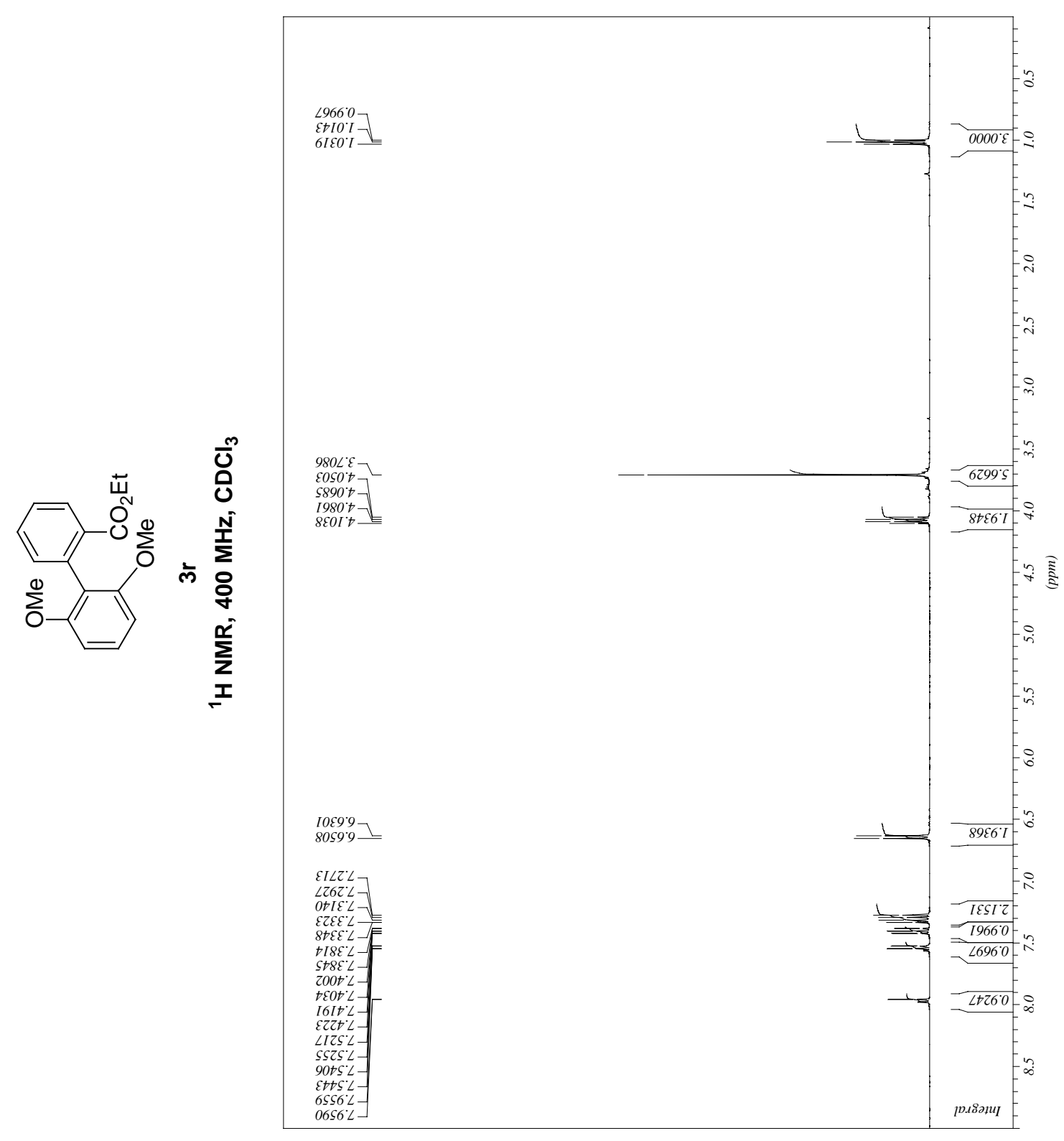




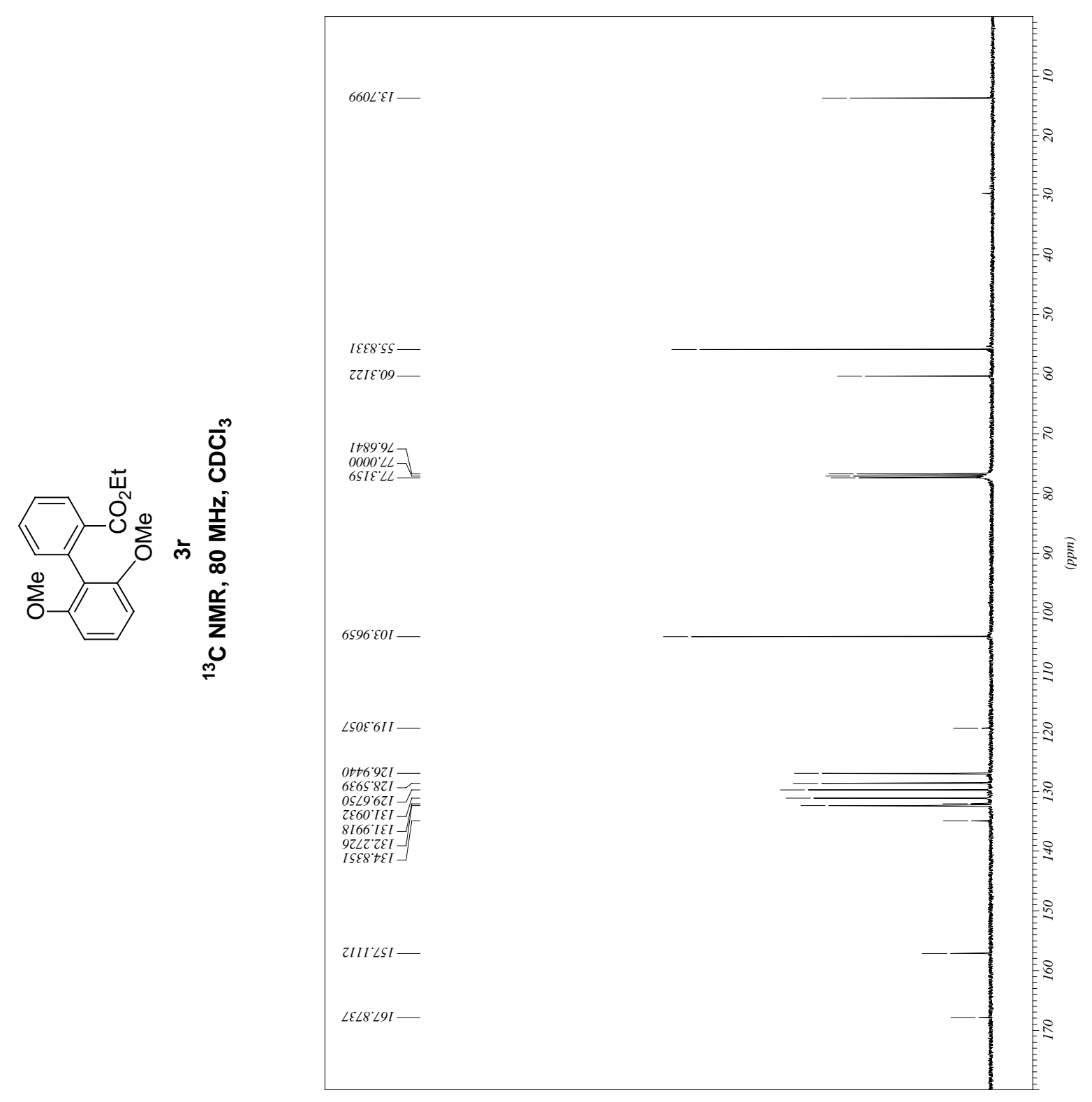




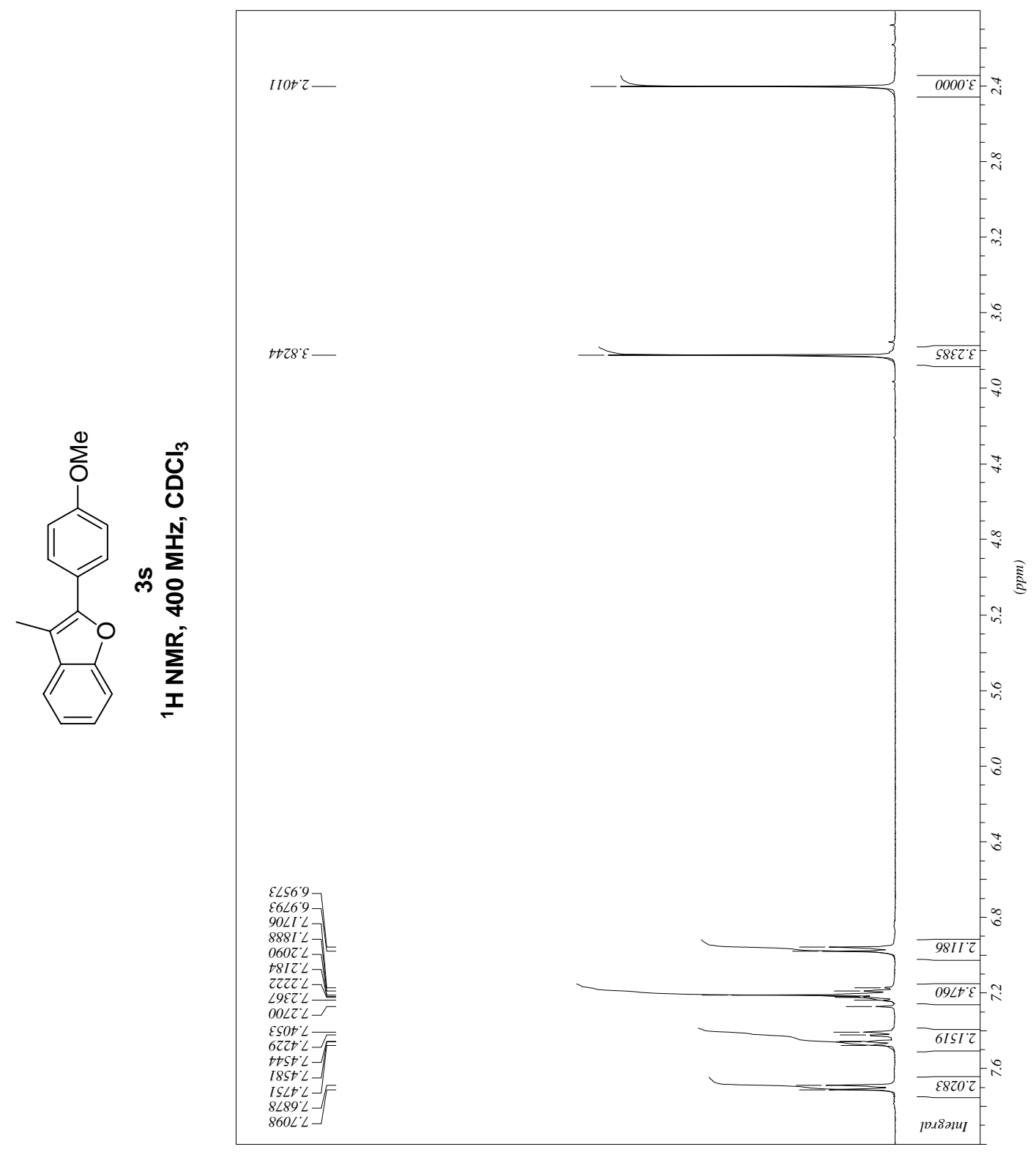




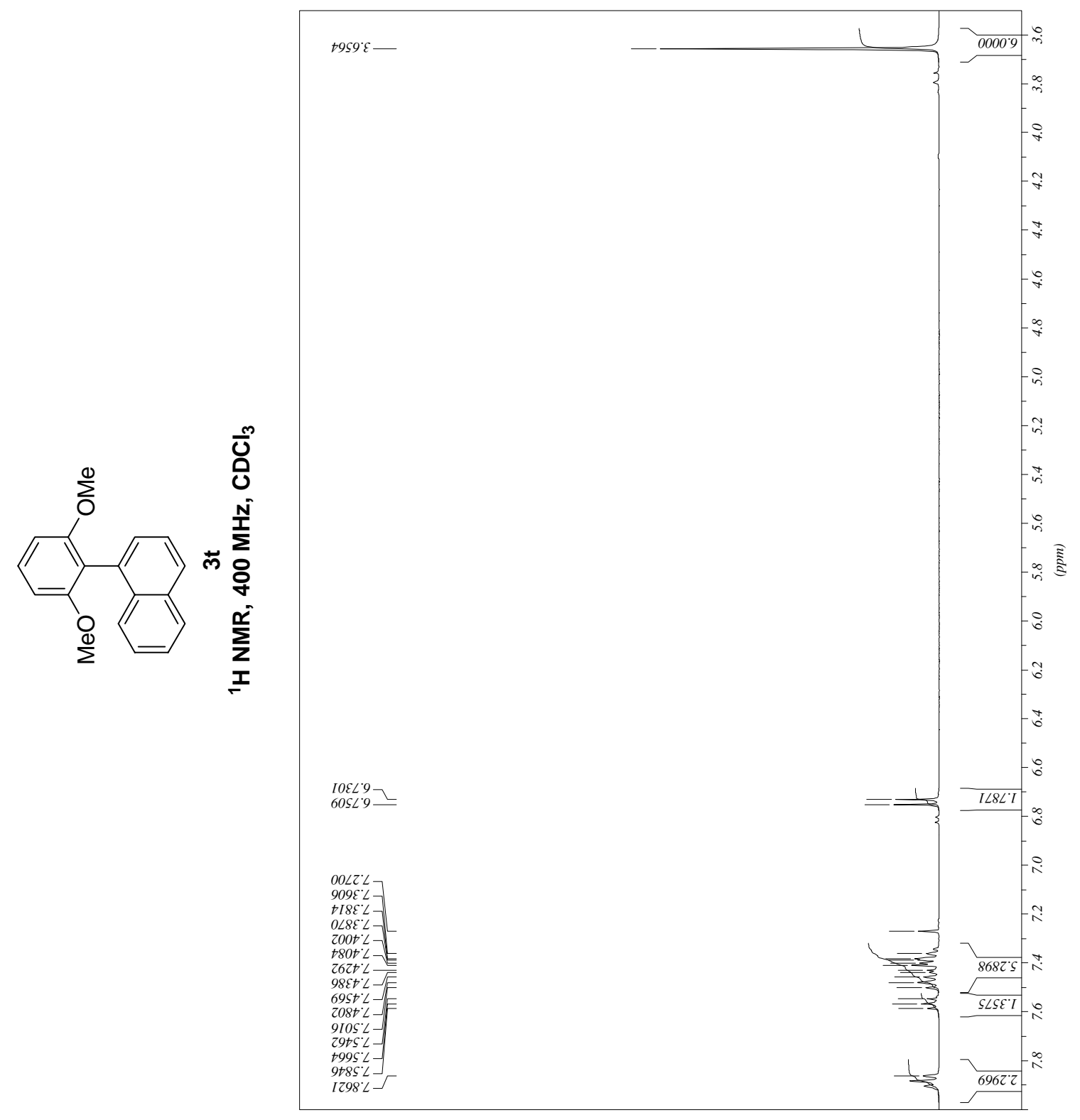




\section{References}

(1) Florvall, L.; Ögren, S.-O. J. Med. Chem. 1982, 25, 1280.

(2) (a) For compounds 3a and 3m see: Milne, J. E.; Buchwald, S. L. J. Am. Chem. Soc. 2004, 126, 13028. (b) For compound 3b see: Kawano, N. Chem. \& Pharm. Bull. 1959, 7, 698. (c) For compounds 3i, 3j, 3k, and 3o see: Becht, J.-M.; Gissot, A.; Wagner, A.; Mioskowski, C. Chem. Eur. J. 2003, 9, 3209. (c) For compound 3f see: Wang, L.; Zhang, Y.; Liu, L.; Wang, Y. J. Org. Chem. 2006, 71, 1284. (d) For compound 3g see: Korenaga, T.; Kosaki, T.; Fukumura, R.; Ema, T.; Sakai, T. Org. Lett. 2005, 7, 4915. (e) For compound 3n see: Saa, J. M.; Martorell, G.; Garcia-Raso, A. J. Org. Chem. 1992, 57, 678. (f) For compound 3p see: Song, C.; Ma, Y.; Chai, Q.; Ma, C.; Jiang, W.; Andrus, M. B. Tetrahedron 2005, 61, 7438. (g) For compound 3s see: Bach, T.; Bartels, M. Synthesis 2003, 6, 925. (h) For compound 3t see: Kamikawa, T.; Hayashi, T. Tetrahedron, 55, 1999, 3455. 